\title{
The grain size(s) of Black Hills Quartzite deformed in the dislocation creep regime
}

\author{
Renée Heilbronner and Rüdiger Kilian \\ Department of Environmental Sciences Geological Institute, Bernoullistrasse 32, 4056 Basel, Switzerland \\ Correspondence to: Renée Heilbronner (renee.heilbronner@unibas.ch)
}

Received: 16 March 2017 - Discussion started: 20 March 2017

Revised: 8 June 2017 - Accepted: 19 July 2017 - Published: 17 October 2017

\begin{abstract}
General shear experiments on Black Hills Quartzite (BHQ) deformed in the dislocation creep regimes 1 to 3 have been previously analyzed using the CIP method (Heilbronner and Tullis, 2002, 2006). They are reexamined using the higher spatial and orientational resolution of EBSD. Criteria for coherent segmentations based on $c$-axis orientation and on full crystallographic orientations are determined. Texture domains of preferred $c$-axis orientation (Y and B domains) are extracted and analyzed separately. Subdomains are recognized, and their shape and size are related to the kinematic framework and the original grains in the BHQ. Grain size analysis is carried out for all samples, high- and low-strain samples, and separately for a number of texture domains. When comparing the results to the recrystallized quartz piezometer of Stipp and Tullis (2003), it is found that grain sizes are consistently larger for a given flow stress. It is therefore suggested that the recrystallized grain size also depends on texture, grain-scale deformation intensity, and the kinematic framework (of axial vs. general shear experiments).
\end{abstract}

\section{Dedication}

This contribution is dedicated to Jan Tullis, whose superb work on experimental rock deformation and microstructure analysis continues to be an inspiration to us all.

\section{Introduction}

Black Hills Quartzite (BHQ) has been used extensively in experimental rock deformation. Coaxial and general shear experiments have been carried out, for example, to define the dislocation creep regimes of quartz (Hirth and Tullis, 1992), to derive flow law parameters (Gleason et al., 1993), to determine the effect of annealing (Heilbronner and Tullis, 2002; Kidder et al., 2016) and the effect of the chemical environment on deformation processes (Post et al., 1996; Chernak et al., 2009), to compare deformation processes to nature (Stipp and Kunze, 2008), and to study the development of texture and microstructure with strain (Tullis et al., 1973; Tullis, 1977; DellAngelo and Tullis, 1989; Heilbronner and Tullis, 2006). BHQ was also used to determine the widely used recrystallized quartz grain size piezometer of Stipp and Tullis (2003). Among the microstructure analyses that were performed in those original papers, grain size was usually determined using CIP misorientation images. However, the CIP method (computer-integrated polarization microscopy; details in Heilbronner and Barrett, 2014) is only capable of detecting the $c$-axis orientation of optically uniaxial materials and hence is only capable of detecting grain boundaries between grains that differ in $c$-axis orientation. One of the puzzling results found by Heilbronner and Tullis (2006) was that the recrystallized grain size seemed to depend on the crystallographic preferred orientation of the grains within a domain. In other words, the grain size seemed to depend not only on the flow stress, but also on the orientation of the $c$ axis with respect to the kinematic framework. At the time, no EBSD analysis (electron backscatter diffraction) was carried out, and hence the full crystallographic orientation was not known. In principle it is therefore possible that some grain boundaries were missed (between grains with paral- 
Table 1. Mechanical data for general shear experiments of Black Hills Quartzite.

\begin{tabular}{|c|c|c|c|c|c|c|c|c|c|c|c|c|c|c|c|c|}
\hline 1 & 2 & 3 & 4 & 5 & 6 & 7 & 8 & 9 & 10 & 11 & 12 & 13 & 14 & 15 & 16 & 18 \\
\hline Regime & Sample & $\begin{array}{r}T \\
{\left[{ }^{\circ} \mathrm{C}\right]}\end{array}$ & {$\left[10^{-5} \mathrm{~s}^{-1} \dot{\gamma}_{\min }\right.$} & {$\left[10^{-5} \mathrm{~s}^{-1}\right]$} & $\begin{array}{r}P \\
{[\mathrm{GPa}]}\end{array}$ & $\begin{array}{r}\mathrm{H}_{2} \mathrm{O} \\
{[\mathrm{wt} \%]}\end{array}$ & $\begin{array}{r}\tau_{\text {peak }} \\
{[\mathrm{MPa}]}\end{array}$ & $\begin{array}{r}\tau_{\text {flow }} \\
{[\mathrm{MPa}]}\end{array}$ & $\begin{array}{r}\tau_{\text {last }} \\
{[\mathrm{MPa}]}\end{array}$ & $\begin{array}{r}\Delta \sigma \\
{[\mathrm{MPa}]}\end{array}$ & $\begin{array}{l}45^{\circ} \mathrm{d} . \\
{[\mathrm{mm}]}\end{array}$ & $\begin{array}{r}\mathrm{th}_{0} \\
{[\mathrm{~mm}]}\end{array}$ & $\begin{array}{r}\mathrm{th}_{\mathrm{f}} \\
{[\mathrm{mm}]}\end{array}$ & $\gamma_{\mathrm{a}}$ & $\gamma_{\mathrm{eff}}$ & $\gamma_{\text {true }}$ \\
\hline $1 \mathrm{a}$ & w940 & 850 & 1.46 & 2.5 & 1.5 & - & 413 & 238 & 238 & 476 & 3.6 & 1.27 & 0.87 & 4.1 & 2.8 & 2.7 \\
\hline $1 b$ & w1092 & 850 & 0.5 & 2.43 & 1.55 & - & 647 & 314 & 338 & 628 & 5.05 & 1.45 & 0.88 & 5.7 & 3.5 & 3.3 \\
\hline $2 a$ & w1086 & 875 & 1.33 & 2.6 & 1.58 & - & 269 & 300 & 294 & 600 & 2.49 & 1.14 & 0.87 & 2.9 & 2.2 & 2.2 \\
\hline $2 b$ & w946 & 875 & 1.04 & 3.08 & 1.5 & 0.17 & 220 & 201 & 265 & 402 & 4.44 & 1.24 & 0.65 & 6.8 & 3.6 & 3.3 \\
\hline $3 a$ & w1010 & 915 & 1.53 & 2.13 & 1.55 & 0.17 & - & 115 & 119 & 230 & 2.65 & 1.27 & 1.03 & 2.6 & 2.1 & 2.1 \\
\hline $3 b$ & w935 & 915 & 1.53 & 2.82 & 1.5 & 0.17 & - & 103 & 133 & 206 & 3.87 & 1.27 & 0.69 & 5.6 & 3 & 2.9 \\
\hline $3 \mathrm{c}$ & w965 & 915 & 1.3 & 2.91 & 1.55 & 0.17 & 125 & 107 & 121 & 214 & 5.07 & 1.25 & 0.75 & 6.8 & 4.1 & 3.9 \\
\hline
\end{tabular}

(1) Dislocation creep regimes: 1, 2, 3; a, b, c = low, high, very high shear strain. (2) Sample number, (3) temperature, (4) minimum shear strain rate (at beginning of experiment, calculated from measured shearing and thinning of sample, for constant applied displacement rate), (5) maximum shear strain rate (at end of experiment, calculated as above), (6) confining pressure (confining medium NaCl), (7) amount of water added, (8) shear stress at peak or yield, (9) steady-state shear stress, (10) shear stress at end of experiment, (11) differential stress calculated as $2 \tau_{\text {flow }}$, (12) displacement of forcing block parallel to $45^{\circ}$ precut, (13) thickness of sample at start $\left(\mathrm{th}_{0}\right),(14)$ thickness of sample at end of experiment $\left(\mathrm{th}_{\mathrm{f}}\right),(15)$ apparent shear strain $\gamma_{\mathrm{a}}=\tan (\Psi)$ at end of experiment as indicated on stress-strain plots of shear experiments where

$\Psi=$ shear angle $=\arctan \left(d /\right.$ thf $\left._{\mathrm{f}}\right),(16)$ effective shear strain $\gamma_{\mathrm{eff}}=\tan (\Psi) / k$ where $k=$ th $_{0} /$ th $_{\mathrm{f}},(17)$ true shear strain $\gamma_{\text {true }}=\tan (\Psi) 2 \ln (k) /(k 2-1)$.

lel $c$ axes) and the grain sizes miscalculated. In the same paper, orientation tracking and ACF (autocorrelation function) shape analysis of the so-called "prism" domains with $c$ axes approximately parallel to the $Y$ axis (vorticity axis) of the kinematic framework showed that these domains grow as a function of strain beyond the size of the original BHQ grain size, forming lenticular aggregates that are more elongated and less rotated than the other domains. Together with the extra large grain size, this suggested that they deform at lower stresses than the other domains. In a set of shear experiments on quartz gouge at the brittle-viscous transition (Richter et al., 2016), flow stresses could be calibrated very accurately and EBSD was used to measure the recrystallized grain size. This presented an opportunity to compare the recrystallized grain size of crushed quartz crystals to that of solid quartzite, i.e., the BHQ used in the 2006 experiments. We therefore remeasured those samples (deformed in the dislocation creep regimes 1, 2, and 3) using EBSD data sets to determine the grain size but also, more generally, to repeat, refine, and expand the microstructure and texture analysis of Heilbronner and Tullis (2006). In this study the focus is on the recrystallized grain size with the aim (a) of comparing CIP- and EBSD-derived grain size measurements, (b) confirming or rejecting the notion that the recrystallized grain size depends on texture, (c) reanalyzing the size, shape, and rheology of the so-called "prism" domain, and (d) of checking if the stress dependence of the recrystallized grain size falls on the quartz piezometer of Stipp and Tullis (2003).

\section{Deformation experiments}

The rock deformation experiments that produced the samples analyzed in this study are described in Heilbronner and Tullis (2006). A solid-medium confining pressure apparatus was used, and approximately $1.25 \mathrm{~mm}$ thick slices of Black Hills Quartzite (BHQ) were placed at an angle of $45^{\circ}$ between forcing blocks as shown in Fig. 1. The experiments were run with a confining pressure of approximately $1.5 \mathrm{GPa}$ and an average shear strain rate of approximately $2 \times 10^{-5} \mathrm{~s}^{-1}$ (see Table 1 for details of experimental conditions). Temperatures were 850,875 , and $915^{\circ} \mathrm{C}$ for regimes 1,2 , and 3 , respectively, and $0.17 \mathrm{wt} \% \mathrm{H}_{2} \mathrm{O}$ was added for one of the regime 2 and all of the regime 3 samples. For each regime, one sample was deformed to a relatively low finite shear strain $(2.6<\gamma<4.1)$ and one or two to a relatively high finite shear strain $(5.6<\gamma<6.8)$; see Table 1 . Note that the finite shear strain reported in the experiments refers to a shear strain calculated in one of two ways: either the displacement $(d)$ of the forcing block along the sample boundary divided by the final thickness $\left(\mathrm{th}_{\mathrm{f}}\right)$ of the sample $\left(\gamma=d / \mathrm{th}_{\mathrm{f}}\right)$ or the summed incremental shear strains, which are calculated as the incremental displacement divided by the instantaneous thickness. Neither of these shear strains can be used to calculate a strain ellipse because the flattening component is not properly taken into account This shear strain is numerically larger than the "true shear strain" or the "effective shear strain". Assuming homogeneous general shear, the ratio $k$ of initial to final thickness $\left(\mathrm{th}_{0} / \mathrm{th}_{\mathrm{f}}\right)$ can be used to calculate the effective shear strain $\gamma_{\text {eff }}=\gamma / k$ or the true shear strain $\gamma_{\text {true }}=\gamma \cdot 2 \cdot \ln (k) /(k 2-1)$ (Fossen and Tikoff, 1993); see Table 1 . For this study, the force record is converted to stress using a modified version of the rigS program (Richter et al., 2016), taking into consideration the decreasing overlap of the forcing blocks (ACF correction) and the increasing confining pressure resulting from the compression of the confining medium inside the vessel (32-33 MPa per mm piston advance depending on temperature). The friction correction (as described in Pec et al., 2016) and the stress correction as proposed by Holyoke and Kronenberg (2010) are omitted. Thinning of the sample is assumed to be linear with the axial advancement of the forcing block (see Fig. 2 in Pec et al., 2016). For every time step, the apparent shear strain is calculated as the total displacement of the forcing block at time $t$ along the shear zone divided by the instantaneous thickness of the shear zone at time $t$. The resulting stressapparent shear strain curves reproduce the curves that were originally published by Heilbronner and Tullis $(2002,2006)$ (Fig. 1). 
(a)

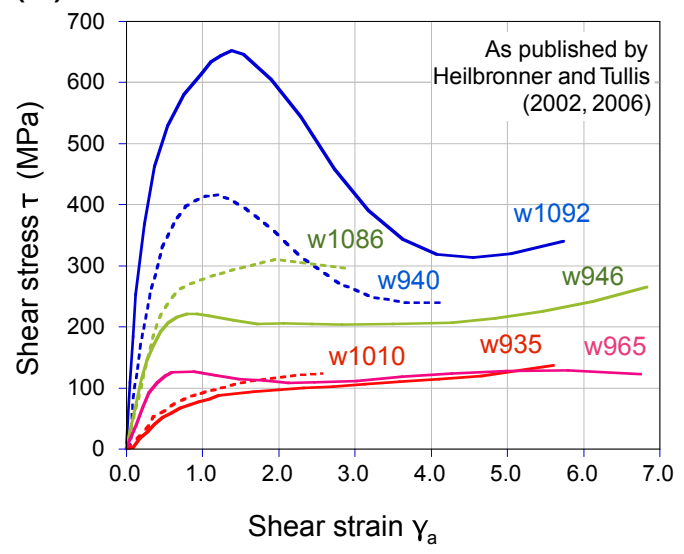

(b)

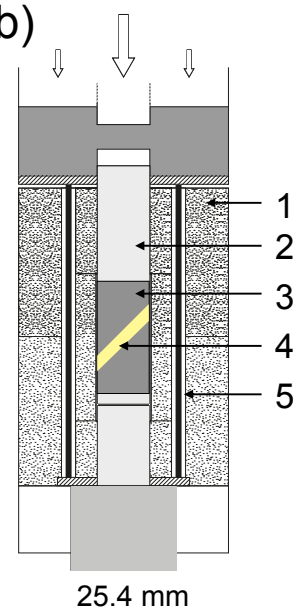

Figure 1. General shear experiments on Black Hills Quartzite. Shear stress $(\tau)$ vs. shear strain $(\gamma)$ : blue is regime 1, green is regime 2 , red is regime 3, the stippled line represents relatively low finite strain, and the solid line represents relatively high finite strain (compare Table 1). On the right, a simplified drawing of the sample assembly for general shear experiments: 1 is the confining medium ( $\mathrm{NaCl}), 2$ is the axial load $/ \sigma_{1}$ piston $\left(\mathrm{Al}_{2} \mathrm{O}_{3}\right), 3$ is the quartz single crystal forcing block, 4 is the quartzite sample at $45^{\circ}$ with respect to $\sigma_{1}$ piston, and 5 is the furnace (carbon, pyrophyllite). Shear strain is an apparent shear strain, and data are recalculated as described in the text.

\section{Data acquisition and processing}

\subsection{Acquisition and preparation of data}

Of each of the deformed samples, a polished thin section of approximately $20 \mu \mathrm{m}$ thickness had been prepared that was suitable for CIP analysis, which was carried out in the previous studies (Heilbronner and Tullis, 2002, 2006). The sections are then polished using Struers Tegramin-30 equipment ( 3 min, $10 \mathrm{~N}$ ) with their MD-Chem neoprene pad and OP-U polishing liquid, which removes at the most a few nm of the sample surface. EBSD data acquisition is carried out using a Zeiss Merlin VP Compact (Zeiss SmartSEM operating software), a NordlysNano camera operated with Oxford AZtec software. Using the settings listed in the Appendix Table A1, maps are acquired at a $1,0.5$, and $0.25 \mu \mathrm{m}$ step size. The data files are exported and the open-source MTEX Toolbox (Hielscher and Schaeben, 2008, https://mtex-toolbox.github. io/) is used for further processing and analysis. If necessary, maps are rotated to have the forcing block in a horizontal direction and flipped such that the shear sense is sinistral for all maps. The maps are exported as Euler RGB maps. Image processing, preprocessing, and analysis is carried out using Image SXM (http://www.liv.ac.uk/ sdb/ImageSXM/), as described in Heilbronner and Barrett (2014). Alternatively and complementary to Image SXM, the open-source software ImageJ (https://imagej.nih.gov/ij/) distributed over the Fiji platform (http://fiji.sc/Fiji) is used. Details of processing and segmentation are described in Appendix A.

\subsubsection{Orientation and misorientation images}

Azimuth and inclination images for the $c$ axes are calculated from EBSD data and displayed as $c$-axis orientation images (COI), which can be viewed with different color look-up tables (CLUTs) depending on the desired feature to be visualized. Using the Spectrum CLUT allows an easy comparison with published COIs obtained by light optical methods (Fig. 2). A so-called AZI CLUT is used to highlight changes in azimuth regardless of inclination (see section on subdomains). Misorientation images (MOIs) are calculated for the $c$-axis orientations. These can be thresholded at a given angle and used as masks to visualize texture domains. Sets of eight misorientation images are used for segmentation with the Lazy Grain Boundaries macro (see Appendix A). Note that in the context of CIP, the term "orientation" refers to $c$-axis orientation (a direction in terms of full texture), and "misorientation" refers to the angle between a given $c$ axis and a reference direction. Note that the maximum value for an angle between two directions (two $c$ axes) is $90^{\circ}$.

\subsubsection{Segmentation}

To decide on the technique for segmentation, automatic and supervised segmentations are performed on three EBSD maps using the method implemented in the MTEX Toolbox (Bachmann et al., 2011) and the Lazy Grain Boundaries macro (Heilbronner and Barrett, 2014), respectively. For the misorientation-angle-based segmentation (EBSD segmentation), which is essentially based on region growing techniques two criteria are used: one considering the full misorientation angle, and the other using the angle between adjacent $c$ axes ( $c$-axis misorientation). The grain sizes result- 


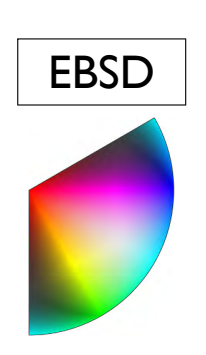

IPFZ
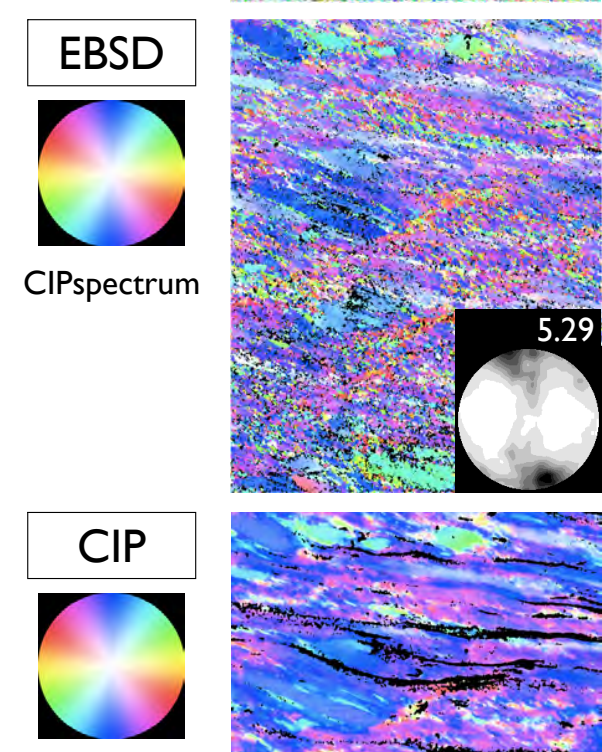

CIPspectrum
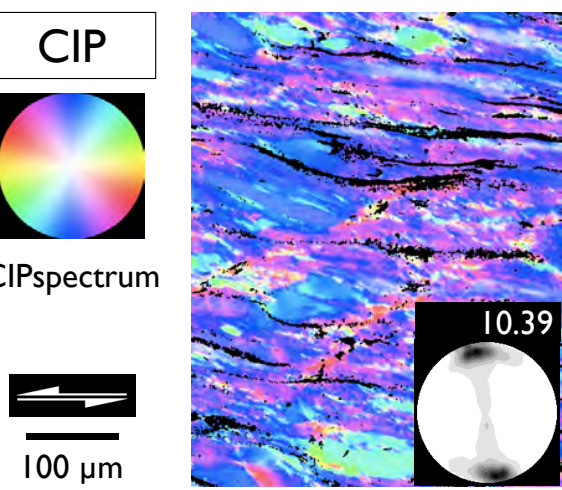
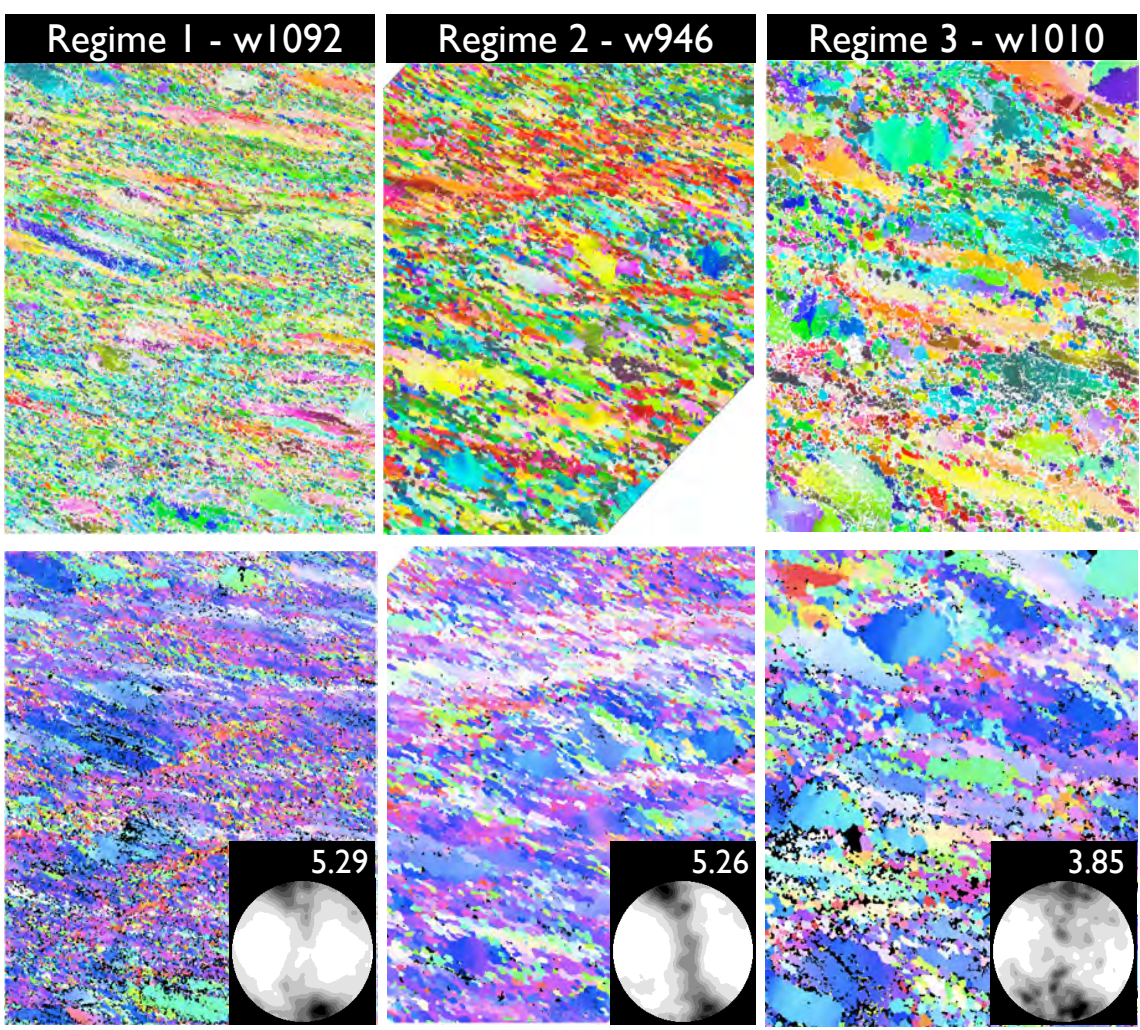
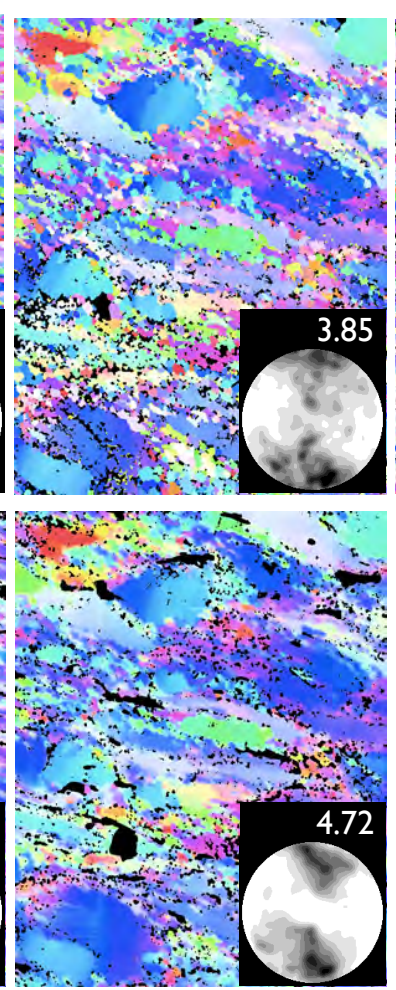
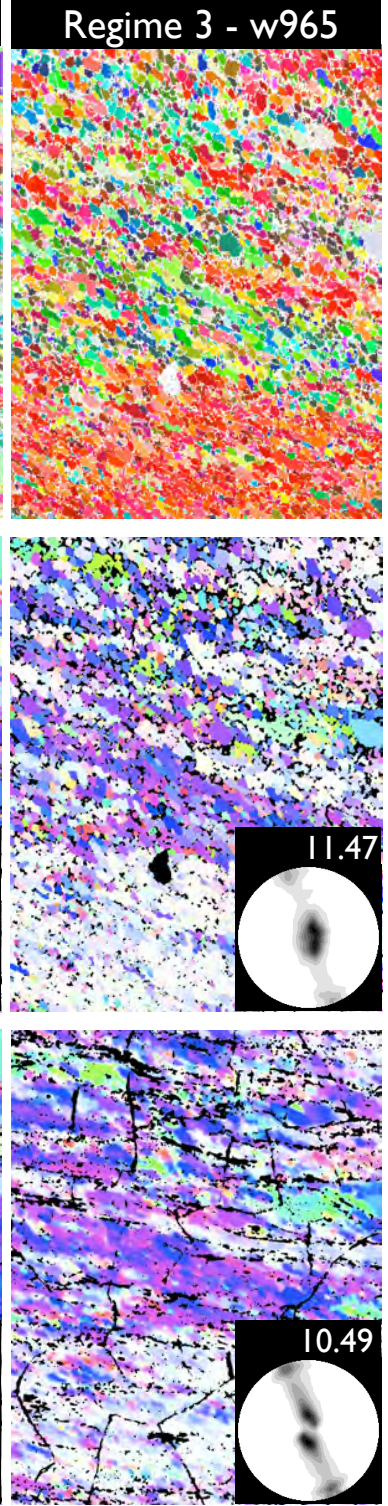

Figure 2. Orientation images. Details of four orientation images are shown with pole figures calculated from total maps. Top row: EBSD maps obtained from SEM using IPFZ (inverse pole figure coloring) for the $z$ direction (image normal). Middle row: Same maps as in row above using CIPspectrum, i.e., LUT for $c$-axis orientation; $c$-axis pole figures calculated from EBSD-derived ODF with maximum indicated above. Bottom row: CIP maps (obtained by polarization microscopy) using same CIPspectrum, i.e., LUT for $c$-axis orientation as row above; $c$-axis pole figures calculated from CIP-derived azimuth and inclination images with maximum indicated above. Scale bar and sinistral shear sense apply to all.

ing from these segmentations are then compared to the grain size derived from the classical Lazy Grain Boundaries (LGB) segmentation, which is based on edge detection and includes structural filtering, i.e., shape aspects. Based on visual comparison of the derived grain boundary maps with the original EBSD maps and in order to perform coherent segmentations for all grain sizes, the LGB segmentation is used throughout this study. See Appendix A for details on the techniques.

\subsubsection{Grain size mapping}

From segmented orientation images, grain size maps are derived (Heilbronner and Barrett, 2014). The grain map is scaled spatially with the scale being determined by the EBSD step size. For each grain, the diameter of the area equivalent circle is calculated. Rejecting grains with an indexing ratio below $75 \%$, the pixels of every valid grain are assigned a gray value $(\mathrm{GV})$ corresponding to the value of the diameter. A cutoff value can be selected and the rainbow LUT is used 
Table 2. Grain size measurements.

\begin{tabular}{|c|c|c|c|c|c|}
\hline 1 & 2 & 3 & 4 & 5 & 6 \\
\hline & & \multicolumn{2}{|l|}{ 3-D diameter } & \multicolumn{2}{|c|}{ 2-D diameter } \\
\hline Map & No. of grains & Mode $(=$ mean $)$ of $v(D)$ & $\mathrm{SD}$ of $v(D)$ & RMS of $h(d)$ & Mean of $h(d)$ \\
\hline \multicolumn{6}{|c|}{ Grain size of undeformed Black Hills Quartzite from 2-D grain size $(0<d \leq 150 \mu \mathrm{m})-$ Fig. 4} \\
\hline BHQ undef. EBSD data & 216 & 101.6 & 5.3 & 77.2 & 70 \\
\hline BHQ undef. CIP data & 1146 & 101.5 & 13.8 & 90 & 86.5 \\
\hline \multicolumn{6}{|c|}{ Recrystallized grain size from 2-D grain size $(0<d \leq 25 \mu \mathrm{m})-$ Fig. 9} \\
\hline 1a-w940 & 5914 & 5 & 2 & 4.1 & 3.6 \\
\hline 1b-w1092 & 34115 & 3.8 & 1.4 & 3.4 & 3 \\
\hline 2a-w1086 & 4377 & 5.5 & 2.2 & 5 & 4.1 \\
\hline 2b-w946 & 19279 & 6.3 & 3 & 4.5 & 3.8 \\
\hline 3a-w1010-s34+s36 & 12233 & 8.9 & 3.4 & 8.2 & 6.7 \\
\hline 3b-w935 & 13354 & 14.7 & 7 & 9.4 & 8 \\
\hline $3 c-w 965-s 40$ & 10910 & 10.7 & 4.4 & 7.6 & 6.6 \\
\hline \multicolumn{6}{|c|}{ Recrystallized grain size of texture domains from 2 -D grain size $(0<d \leq 50 \mu \mathrm{m})$} \\
\hline 1b-w1092 & 34115 & 3.7 & 1.4 & 3.4 & 3 \\
\hline 1b-w1092 B domain & 11647 & 4.2 & 1.8 & 3.5 & 3.1 \\
\hline 1b-w1092 Y domain & 2289 & 3.6 & 1.3 & 3.3 & 2.9 \\
\hline 2b-w946 & 19279 & 6.1 & 2.9 & 4.5 & 3.8 \\
\hline 2b-w946 B domain & 7425 & 6.7 & 3.4 & 4.8 & 4 \\
\hline 2b-w946 Y domain & 5634 & 6.1 & 2.8 & 4.4 & 3.8 \\
\hline 3b-w935 & 13354 & 14.2 & 6.8 & 9.4 & 8 \\
\hline 3b-w935 B domain & 2817 & 13.7 & 6.9 & 9.2 & 7.8 \\
\hline 3b-w935 Y domain & 7702 & 14.8 & 7.1 & 9.6 & 8.1 \\
\hline 3c-w965 & 10910 & 10.5 & 4.3 & 7.6 & 6.6 \\
\hline 3c-w965 B domain & 2203 & 11 & 4.8 & 7.8 & 6.8 \\
\hline 3c-w965 Y domain & 7385 & 10.6 & 4.3 & 7.6 & 6.7 \\
\hline
\end{tabular}

(1) Processed maps: prefixes 1,2, 3 indicate regimes 1,2, 3; a, b, c $=$ low, high, very high shear strain. B domain, pixels with $c$-axis maximum at periphery of pole figure; $\mathrm{Y}$ domain, pixels with $c$-axis maximum in center of pole figure. (2) Number of grains with $>75 \%$ of pixels indexed, (3) mode of $v(D)$ where $v=$ volume weighted distribution and $D=$ diameter of recalculated 3-D grains (volume equivalent spheres) = mean of Gaussian fit, (4) SD of Gauss fit, (5) root mean square of frequency distribution of diameter of 2-D sections $h(d)$ where $h=$ number density and $d=$ diameter of 2-D grains (area equivalent circles), (6) mean of frequency distribution of diameter of 2-D sections $h(d)$.

to visualize small values in blue and values above the cutoff in red (Fig. 3). Note that grain size maps are both scaled in $X$ and $Y$ (spatial coordinates of the image plane) and calibrated in $Z$ (gray values).

\subsection{Grain size analysis}

\subsubsection{Determination of 2-D grain size}

The 2-D diameter of each segment is calculated from the cross-sectional area. The number-weighted distribution $h(d)$ of area equivalent diameters is presented as a histogram, for which the arithmetic mean, the mode, etc. can be determined. In order to be able to plot the data on the piezometer of Stipp and Tullis (2003), the root mean square (RMS) is calculated. Note that the RMS is biased towards the upper end of the distribution (larger grain sizes) and returns a value greater than the arithmetic mean. Because the RMS depends strongly on the tail end of the distribution, the histogram has to be cropped carefully to the relevant size range if the RMS is to be a meaningful measure of the grain size.

\subsubsection{Converting to 3-D}

To obtain a possible parent distribution of 3-D grains, the program StripStar (Fortran source stripstarD.f and Matlab script stripstar.m; see the Supplement) is used (details in Heilbronner and Barrett, 2014). The mode of the volumeweighted histogram of 3-D diameters, $v(D)$, is found by fitting a Gaussian with the mean of the Gaussian representing the mode of $v(D)$ (Fig. 4). Note that the mean is centered about the mode of $v(D)$ and is therefore independent of the long tail end of the distribution. In many instances, volume weighting is considered to be physically more meaningful than number weighting because it is the mass of a certain grain size fraction that matters, not the number of grains in it. An alternative is to use a lognormal fit as suggested by 

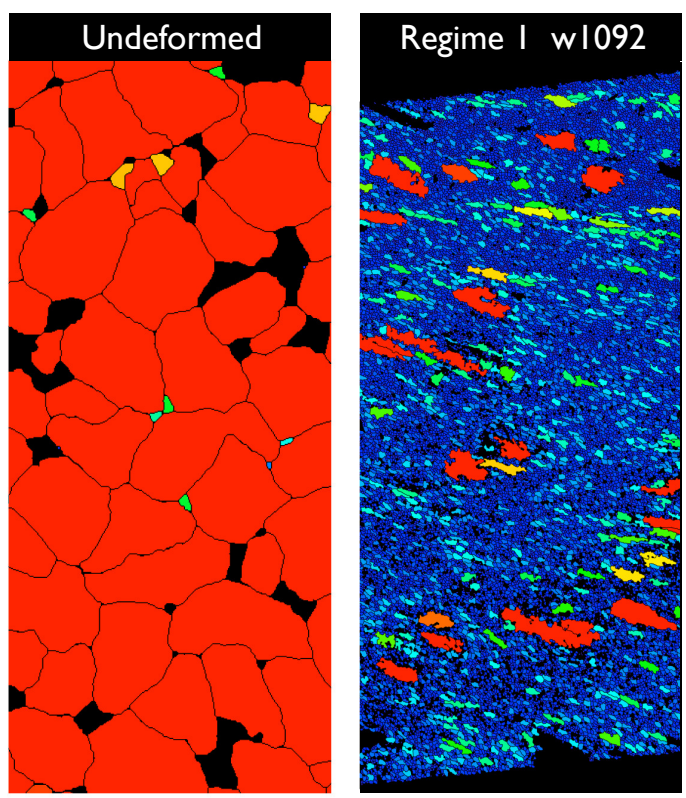

Area equivalent diameter $(\mu \mathrm{m})$

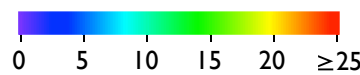

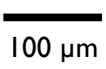
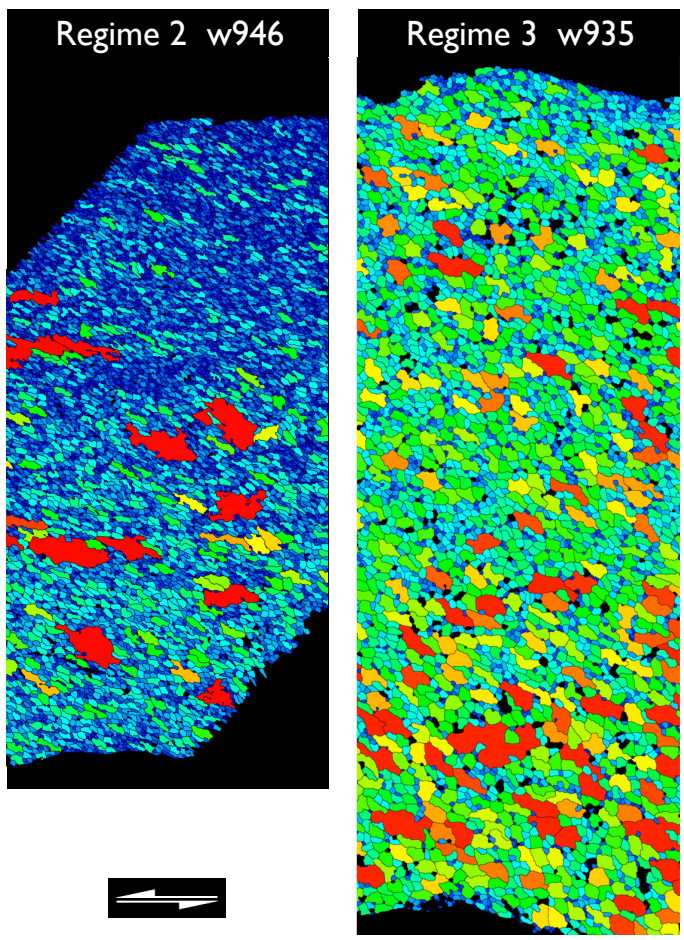

Figure 3. Grain size maps. Color-coded grain size maps visualizing the diameter of area equivalent circles. From left to right: for undeformed Black Hills Quartzite and samples deformed in regimes 1, 2, and 3. Scale, shear sense, and look-up table for grain size apply to all. Red indicates the diameter of an area equivalent circle $d \geq 25 \mu \mathrm{m}$. Note that the diameter of undeformed Black Hills Quartzite is $\sim 100 \mu \mathrm{m}$.

Lopez-Sanchez and Llana-Funez (2016); see Appendix A. A Gaussian normal fit usually results in a slight overestimation and a lognormal fit in a slight underestimation of the mode compared to a kernel density estimate. The advantage of normal and lognormal fits is that statistically meaningful parameters can be derived. All 3-D and 2-D grain sizes evaluated for this study, i.e., the modal values of $v(D)$ and the RMS of $h(d)$, are determined by fitting a Gaussian; they are listed in Table 2. In the following, the term "3 mode" will refer to the mode of $v(D)$ and the term "2-D RMS" to the RMS of $h(d)$, representing measures of 2-D and 3-D grain size, respectively.

\subsection{Extracting texture components}

Here, the concept of a "texture component" is not based on the full crystallographic information, i.e., not defined by all three Euler angles; instead it refers to aspects of $c$-axis orientation only. To construct a domain map of a given texture component, the $c$-axis misorientation image (MOI) is used. This image is thresholded at a level corresponding to the desired opening angle about the reference direction of the MOI, which is identified on the pole figure, i.e., about the central $c$-axis orientation of the domain. Each of the domain maps shown in Fig. 5 is created by superposing a mask made from the MOI thresholded at $15^{\circ}$ (for a $30^{\circ}$ opening angle) on the $c$-axis orientation image with a continuous color coding of $360^{\circ}$ of azimuth (AZI CLUT). In the paper by Heilbronner and Tullis (2006), a number of $c$-axis maxima and domains were identified. Their names allude to the slip system, the activation of which was supposed to give rise to them (Bouchez and Pecher, 1981; Schmid and Casey, 1986). The "prism" domain with $c$ axes parallel to the structural $Y$ direction is interpreted to result from prism $\langle a\rangle$ slip; the "basal" domain with $c$ axes on the periphery slightly rotated from the structural $Z$ direction in the sense of shear is interpreted to be related to basal $\langle a\rangle$ slip; the "rhomb" domain with two symmetrically disposed $c$-axis maxima on the inclined girdle, suitably oriented for rhomb $\langle a\rangle$ slip; and the $\sigma_{1}$ domain with a $c$-axis maximum on the periphery oriented in the "hard" direction, i.e., parallel to the direction of the applied load. Here, the names for the domains are Y domain, B domain, $\mathrm{R}$ domain, and $\sigma_{1}$ domain, respectively (see Fig. 5, inset lower right), but without implicitly assuming that a specific $c$-axis orientation implies the activity of a certain slip system.

\subsection{Maps of misorientation density}

To investigate the conspicuous grain size gradient of the regime 1, 2, and 3 samples shown in Fig. 3, the grainaveraged kernel average misorientation (gKAM) is determined by a method described in detail in the companion paper (Kilian and Heilbronner, 2017, this volume) and mapped 
as shown in Fig. 6. Briefly, a higher-order kernel average misorientation (KAM) is calculated on orientation-noisereduced EBSD data. For each grain, the sum of the KAM is divided by the number of measurements, providing the gKAM, which is a measure of the misorientation density and related to the intragranular deformation intensity.

\subsection{Shape and spatial analysis}

To analyze the shape and size of the texture domains and subdomains, the autocorrelation function (ACF) is used. To determine spatial clustering, the internal grain boundaries and the subdomain boundaries are subjected to a contact area analysis (Lazy Contacts macro). Both of these methods are described in Heilbronner and Barrett (2014).

\section{Results}

\subsection{Automatic vs. supervised segmentation}

For the three high-strain samples of regimes 1, 2, and 3 (w1092, w946, w935), segmentations with increasing misorientation angles up to $15^{\circ}$ are checked against the corresponding LGB segmentation. Segmentation based on full misorientation angles (solid lines) and on $c$-axis misorientation angles (stippled lines) show very similar behavior. To achieve the same 3-D mode of grain size for both techniques, the threshold misorientation angles for segmentation have to be 2.5 and $4.5^{\circ}$ (regime 1 ), 3 and $3.5^{\circ}$ (regime 2), and 5.6 and $5.7^{\circ}$ (regime 3 ). Similar values also result for the 2-D RMS and the 2-D mean grain size in regimes 2 and 3. For regime 1, the 2-D RMS and the 2-D mean never reach the value attained by the LGB segmentation (Fig. 7).

\subsection{Grain size of dynamic recrystallization regimes}

The 2-D grain size distribution is visualized using grain size maps (Fig. 3). A few aspects in this figure merit attention. Comparing the predominant colors of the grain size maps with this CLUT suggests that the cross-sectional areas of most of the grains of the regime 1 samples have diameters less than $5 \mu \mathrm{m}$, those of regime 2 less than $8 \mu \mathrm{m}$, and those of regime 3 less than $15 \mu \mathrm{m}$. In addition, a rather clear grain size gradient can be recognized for w946. Expressed in terms of the 3-D mode, the grain size of the starting material (undeformed BHQ) is $101 \mu \mathrm{m}$, which is much larger than the recrystallized grain size (Fig. 4, inset). The recrystallized grain size for the seven samples deformed in regimes 1,2, and 3 to high and low total shear strain is shown in Fig. 4. The modal values found for the samples w1092, w946, and w935 shown as grain size maps in Fig. 3 are 3.7, 6.1, and $14.2 \mu \mathrm{m}$, respectively, which are values that coincide with the visual impressions of $<5,<8$, and $<15 \mu \mathrm{m}$. Note that the corresponding RMS values of 3.4, 4.5, and $9.4 \mu \mathrm{m}$ (Table 2) do not fit the visual impression as nicely. The high-strain samples are al- most completely recrystallized. The same is not true for the low-strain samples, and accordingly their distributions $v(D)$ are not strictly monomodal but show relatively high volume fractions at the larger end of the histogram.

\subsection{Identification of subdomains}

Upon closer inspection, the pole figures reveal that the maxima of the Y- and B-texture components are usually composed of two distinct submaxima. Selecting these ("upper" and "lower") submaxima in a pole figure, two separate orientation images for the corresponding texture component, i.e., two subdomains, can be created (Fig. 5). What was originally considered one $\mathrm{Y}$ or one $\mathrm{B}$ domain is actually composed of two non-intersecting spatial domains as evidenced by the different colors, which highlight the distinct ranges of the azimuth of $c$-axis orientations of each of the subdomains.

\subsection{Grain size and misorientation density}

To explore the relation between grain size and the state of deformation (as indicated by misorientation density), the grain maps are evaluated separately for high- and low-gKAM regions (Fig. 6). The 3-D modes are determined for the upper and lower halves of the samples w1092 (regime 1) and w946 (regime 2) and in four strips of sample w935. Clearly, regions of higher gKAM have a smaller recrystallized grain size and regions of lower gKAM have a larger recrystallized grain size. Large, non-recrystallized grains may also have high gKAM values but are not considered in this analysis. The correlation between the 2-D RMS and the gKAM is also evident from the continuous, shear zone boundary parallel traverse through sample w946 (Fig. 6).

\subsection{Grain size of domains and subdomains}

The grain size analysis for the Y domain of sample w935 (regime 3) and its subdomains is visualized in Fig. 8. The histograms denoted by 2-D are obtained by grouping the gray values (which are calibrated to the 2-D diameter of the grains). The mean value of the histograms represents the arithmetic mean of the area-weighted size distribution $\mu_{\mathrm{a}}(d)$. This means, for example, that in the case of the $\mathrm{Y}$ domain of w935, the mean area fraction is occupied by grains $14.2 \mu \mathrm{m}$ in diameter, i.e., a $158 \mu \mathrm{m}^{2}$ cross-sectional area. The 3-D mode is shown with histograms denoted 3-D. Comparing $\mu_{\mathrm{a}}(d)$ with the 2-D RMS and the 3-D mode, one finds that 2-D RMS $<\mu_{\mathrm{a}}(d)<3$-D mode (see also Table 2 ). The area-weighted distribution of diameters is not to be confused with the frequency distribution of areas, which for the same domain has an arithmetic mean of $247 \mu \mathrm{m}^{2}$ corresponding to a diameter of $17.7 \mu \mathrm{m}$.

The grain sizes of the (combined) B and $\mathrm{Y}$ domains have been calculated for the high-strain samples in regimes 1, 2, and 3 (Fig. 9). The ratio between the recrystallized grain size of the Y domains and the bulk grain size is $>1.00$ for sam- 

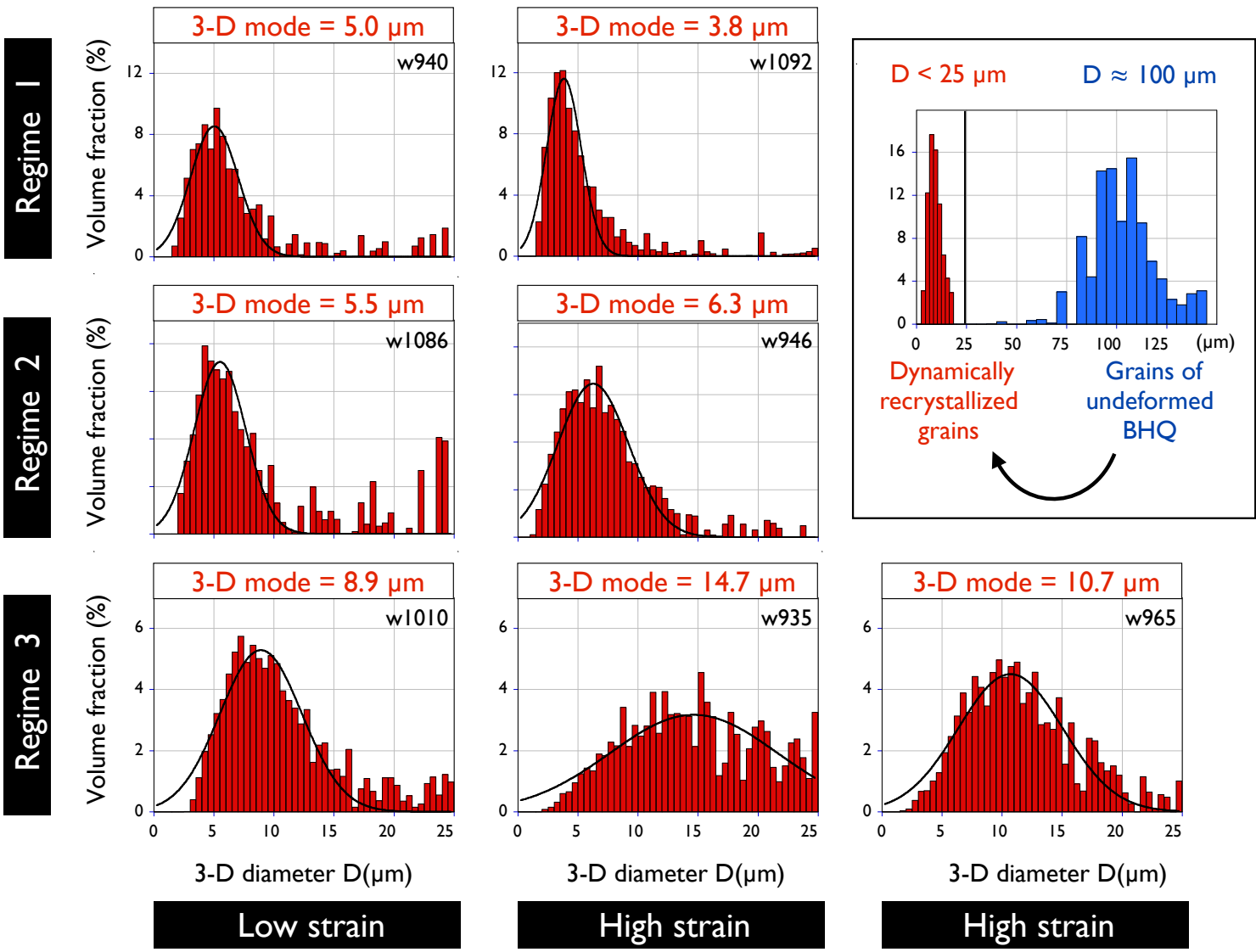

Figure 4. Recrystallized grain size for dislocation creep regimes 1, 2, and 3. Volume-weighted histograms $v(D)$ are shown for seven samples for relatively low $(2.7<\gamma<4.3)$ and high shear strains $(5.8<\gamma<7.1)$. $D$ is the diameter of volume equivalent sphere. The mode of $v(D)$ is obtained by a Gaussian normal fit to the distribution. Inset shows the grain size distribution of undeformed and deformed Black Hills Quartzite for comparison.

ple w935, $\approx 1.00$ for samples w965 and w946, and $<1.00$ for sample w1092. Conversely, the ratio between the recrystallized grain size of the $\mathrm{B}$ domains and the bulk grain size is $>1.00$ for samples w965, w946, and w1092 and $<1.00$ only for sample w935 (see also Table 2).

\subsection{Grain size and flow stress}

The grain size data are plotted on the piezometer of Stipp and Tullis (2003). The results are presented in two types of plots in Fig. 10: in the top row, 3-D modes are used for the diameter; in the bottom row, the corresponding 2-D RMS values are plotted to fit the piezometer data set. On account of the high volume fraction of recrystallized grains $(\geq 90 \%)$, the highstrain samples are considered the most reliable data points and are plotted separately. In view of the grain size gradient across the samples w1092, w946, and w935 (see Fig. 3), both the minimum and maximum grain sizes are shown, and the line fit considers all six data points. The picture does not change fundamentally if the low-strain samples are included.
Finally, two slightly different line fits are obtained for the recrystallized grain sizes of the $\mathrm{Y}$ and $\mathrm{B}$ domains.

\section{Discussion}

\subsection{Comparison of CIP and EBSD analysis}

Processing and representing the EBSD mapping as $c$-axis orientation images (COIs) shows that both methods, EBSD and CIP, coincide down to the limit of optical resolution of polarization microscopy (Fig. 2). The maps are very similar, and the geometry of the $c$-axis pole figures are also very similar with differences due to a number of circumstances. Firstly, the SEM penetrates a small layer near the surface of the thin section, whereas the CIP method works in transmission. In optical light microscopy, information from the entire thickness of the thin section contributes to the result, and, even more importantly, grain boundaries appear as a separate phase of isotropic (dark) material and thus disturb the analysis of the nearby pixels, especially if the grain boundaries are orientated at a low angle to the section surface. A sec- 

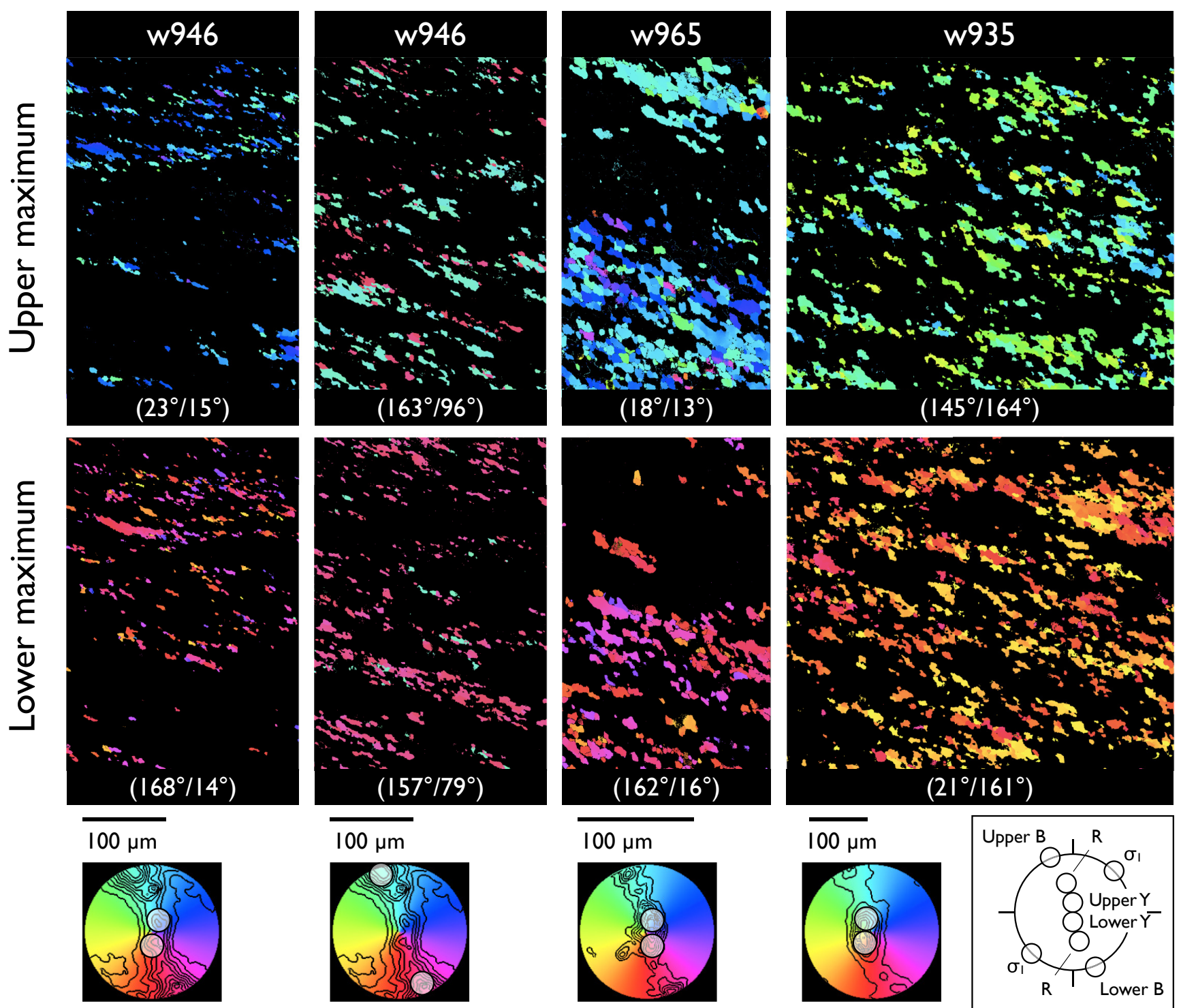

Figure 5. Details of $c$-axis texture. Color-coded orientation images highlighting the double maxima associated with the B and Y domains. Pairs of domain maps are derived for $c$-axis orientations within a $30^{\circ}$ cone $\left(15^{\circ}\right.$ opening angle) with respect to the central orientation. The central directions ( $c$-axis azimuth and inclination) of the submaxima are indicated below the images and correspond to the upper and lower maximum on the pole figure shown at the bottom. Pure azimuth coloring is used for the contoured pole figures and the orientation images. The schematic drawing at the lower right includes preferred $c$-axis orientations proposed by Heilbronner and Tullis (2006): B ("basal"), R ("rhomb"), Y ("prism") and the direction of the axial load (at $45^{\circ}$ with respect to the shear zone, usually inferred to be the direction of $\sigma_{1}$ ).

ond source for differences between EBSD and CIP are different procedures by which orientations are calculated from the input, a critical issue being the determination of $c$-axis inclinations in CIP.

The $c$-axis pole figures obtained by the CIP and EBSD methods also compare very well (Fig. 2); the main difference is the level of the pole figure maxima. The comparatively high maximum of the CIP analysis of regimes 1 and 2 may be due to the fact that small recrystallized grains were not resolved, and only relatively few porphyroclasts contributed to the texture. In regime 1, this also accounts for the miss- ing densities on the weak girdle visible on the corresponding EBSD analysis. It came as a surprise that full texture analysis confirms that the so-called basal and prism maxima of $c$ axes (Heilbronner and Tullis, 2006) are actually composed of two distinct submaxima. Previously, when conducting the CIP analysis, it was always considered a problem of not being able to properly calibrate the inclination of the $c$ axes if the B maximum did not appear exactly on the periphery and if the $\mathrm{Y}$ maximum did not occupy one position rather than two at or near the center of the pole figure (Fig. 2, sample w965). However, the misorientation images (Fig. 5) and the 

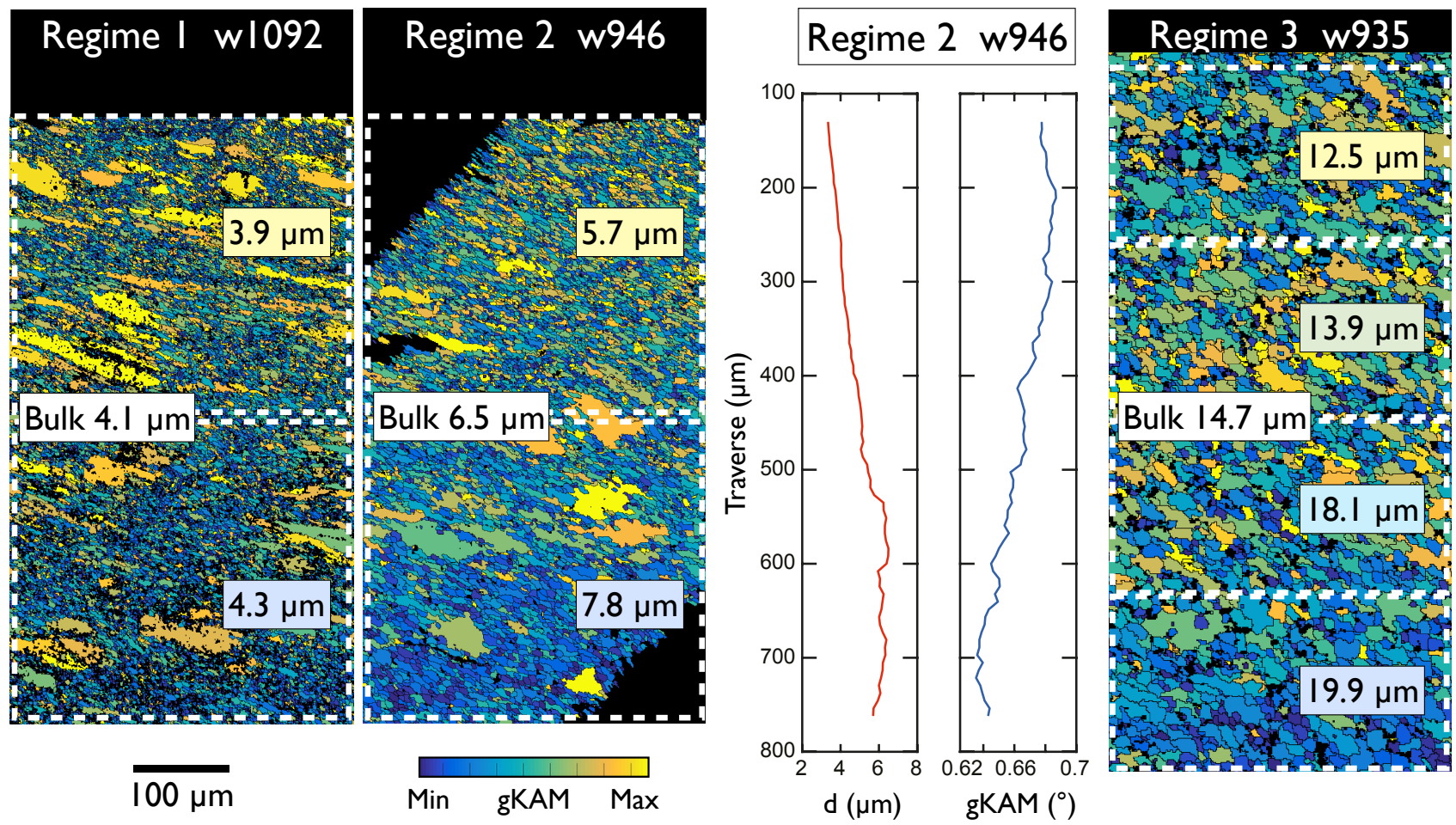

Figure 6. Recrystallized grain size as a function of misorientation density. Maps of grain kernel average misorientation (gKAM) are shown for three dislocation creep regimes. Maps cover nearly the full width of the shear zone. Gradients of gKAM are clearly visible. Modes of $v(D)$ (size distribution of 3-D grains) are calculated for high- and low-gKAM sites in samples w1092 and w946 and for four different sites in sample w935. For sample w946, parallel traverses of grain size (RMS of 2-D equivalent diameters) and gKAM are shown. Scale bar and color coding of gKAM apply to all.

grain size maps (Fig. 8) confirm very clearly that two distinct maxima with corresponding distinct texture domains but similar grain size - do indeed exist.

\subsection{Criteria for grain identification}

For the LGB segmentation, eight misorientation images (MOIs) were used (see Appendix A). On account of the histogram equalization carried out to enhance the contrast in the MOIs, the effective $c$-axis misorientation angle for the definition of a grain boundary is difficult to assess. Comparing the thresholded gradient images to the corresponding orientation gradient images (OGIs) showed that a minimum $c$-axis misorientation angle of 1.2 to $2.5^{\circ}$ defines a grain boundary (see Appendix Table A2). Sometimes this leads to lowangle boundaries being classified as grain boundaries (Appendix Fig. A1, white arrow). The difference between EBSD and LGB segmentations depends on the level of indexing. If a low indexing ratio is due to holes or dust particles, the LGB and EBSDnc (without grain completion) are suitable because they avoid incorporating "foreign phases" into grains (Appendix Fig. A1, black arrow). In these situations, grain completion must be supervised. If low indexing is due to poor pattern quality, however, EBSDc (with grain completion) is probably more suitable because it can merge grains that are dissected by patches of non-indexed pixels. Comparing the three segmentations in Appendix Fig. A1, the first impression is that the grain boundaries of the EBSD and LGB segmentations coincide very well, in particular the EBSDnc segmentation that did not include grain completion is strikingly similar to the LGB segmentation by producing the same holes and gaps.

LGB and EBSD segmentations were tested on a number of samples. The result was always the same regardless of the level of indexing: the resulting grain size of the LGB method was smallest followed by the EBSDnc, and the largest grain size was returned by the EBSDc. Comparative histograms of 2-D diameters of LGB vs. EBSDc and EBSDnc and values for the 2-D RMS and 3-D mode values are shown in Appendix Fig. A1. That grain completion leads to a larger grain size is not surprising, as it allows for the incorporation of non-indexed pixels into the grains. However, the indexing ratio alone cannot account for the differences in segmentation. Another reason for the consistently larger 3-D grain size found by both EBSD methods lies in the cleanup procedure used for the EBSD data, which removes single pixel grains, 

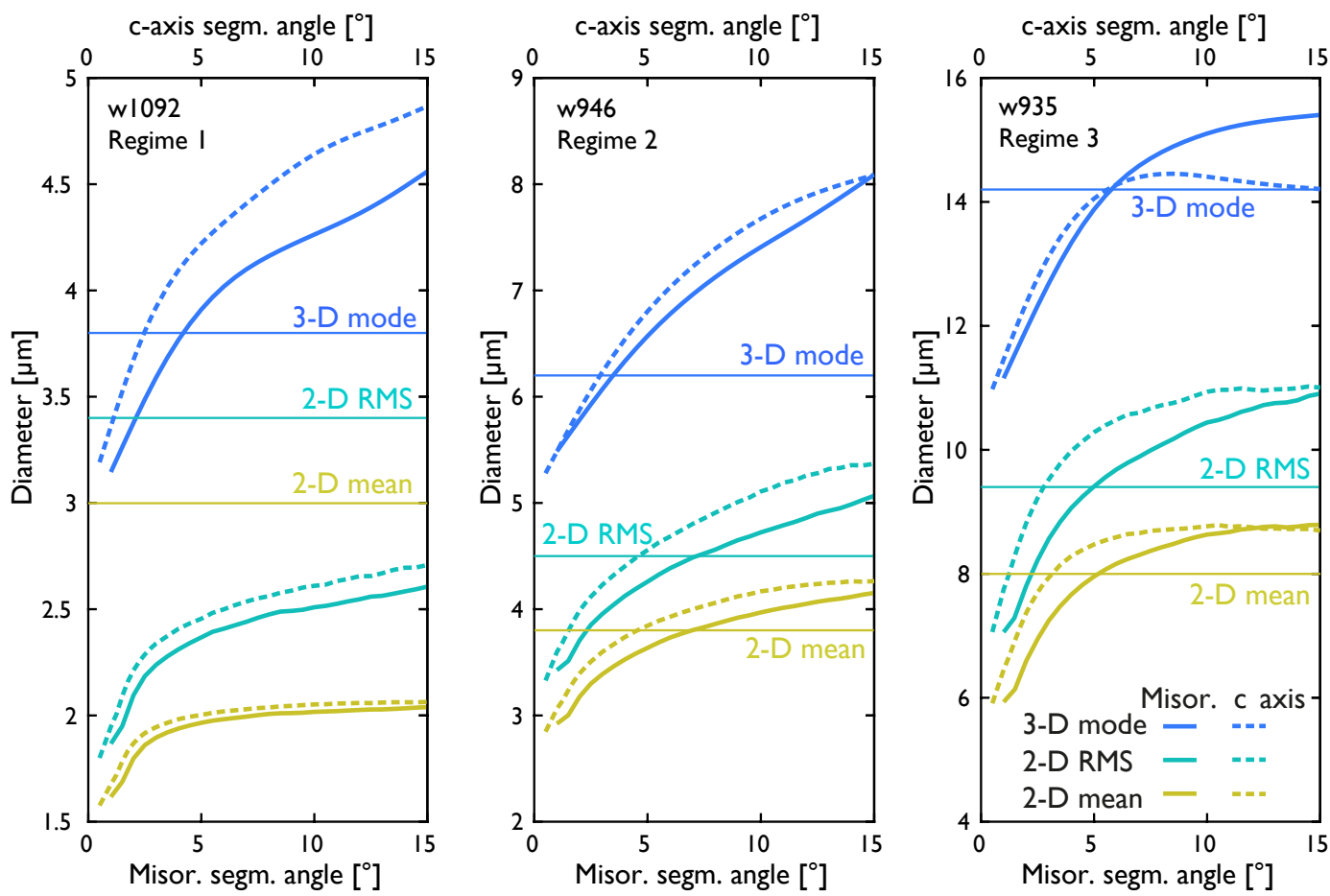

Figure 7. Grain size as a function of segmentation angle. Mean grain sizes calculated without grain completion from full texture data (EBSD maps) using full misorientations (solid lines, bottom $x$ axis) and $c$-axis misorientation (dashed lines, top $x$ axis). Horizontal lines show values obtained by supervised segmentation of CIP images using $c$-axis misorientation images, the Lazy Grain Boundaries macro and the StripStar program for 2-D-3-D conversion. Blue: modes of histograms $v(D)$ of volume fractions of calculated 3-D equivalent diameters; turquoise: RMS of measured 2-D equivalent diameters; yellow: arithmetic mean of measured 2-D equivalent diameters.

leaving the smallest grain size class empty (see, for example, the histograms of w935 in Appendix Fig. A1).

In EBSD segmentation, a misorientation angle of $10^{\circ}$ is typically used. However, EBSD segmentations using full or only $c$-axis misorientation angles show that the misorientation angle that best reproduces the supervised segmentation is much lower. Using an angle of approximately $5^{\circ}$ or less reproduces the CIP/LGB grain sizes; the commonly used misorientation angles of $10^{\circ}$ would overestimate the CIP/LGB grain size considerably (Fig. 7). Note that the CIP/LGB segmentation defines grains as being bounded not only by highangle grain boundaries, but also by low-angle or subgrain boundaries with the transition in quartz being in the range of 9 to $14^{\circ}$ as suggested by Shigematsu et al. (2006). We conclude therefore that the EBSD segmentation based on region growing and CIP/LGB segmentation based on boundary detection yield consistent results while being an alternative concept for the definition of grains.

\subsection{The recrystallized grain size in the dislocation creep regime}

Recrystallized grain size piezometer relations are written as $d=A \sigma^{k}$ or $\log (d)=A+k \log (\sigma)$ (Twiss, 1977; Poirier, 1985 ), where $d$ stands for this average grain size. The recrys- tallized grain size is assumed to be in a steady state during dynamic recrystallization, while it might depend on the recrystallization mechanism or temperature (e.g., Poirier and Guillope, 1979; Shimizu, 2008). However, before exploring any of those dependencies, finding this grain size is not trivial, mostly because what we see of the grain size distribution is a 2-D section of it. One option is to determine the mean grain size from the size distribution of area equivalent circles, and the other is to convert the 2-D circles to 3-D spheres and determine the mean grain size from the size distribution of the spheres. Here, both the 2-D and the 3-D grain sizes were determined, the former because the results can be compared against the piezometer of Stipp and Tullis (2003) and the latter because it depends less on the shape of distribution than the former, thus providing a more reliable measure (Fig. 10).

To assess grain growth during annealing, Heilbronner and Tullis (2002) performed gain size analyses of the same samples that are reanalyzed here. Using a much coarser binning limited by an old version of the StripStar program, they published histograms of $v(R)$, where $v$ is the volumeweighted distribution and $R$ the radius of the volume equivalent spheres. Maximum frequencies of regime 1,2, and 3 samples occurred in the $2-4 \mu \mathrm{m}$ bin (w940), 2-4 um bin (w946), and 6-8 $\mu \mathrm{m}$ bin (w935 and w920), and the estimated modal 3-D diameters were given as 7,8 , and $14 \mu \mathrm{m}$. The val- 

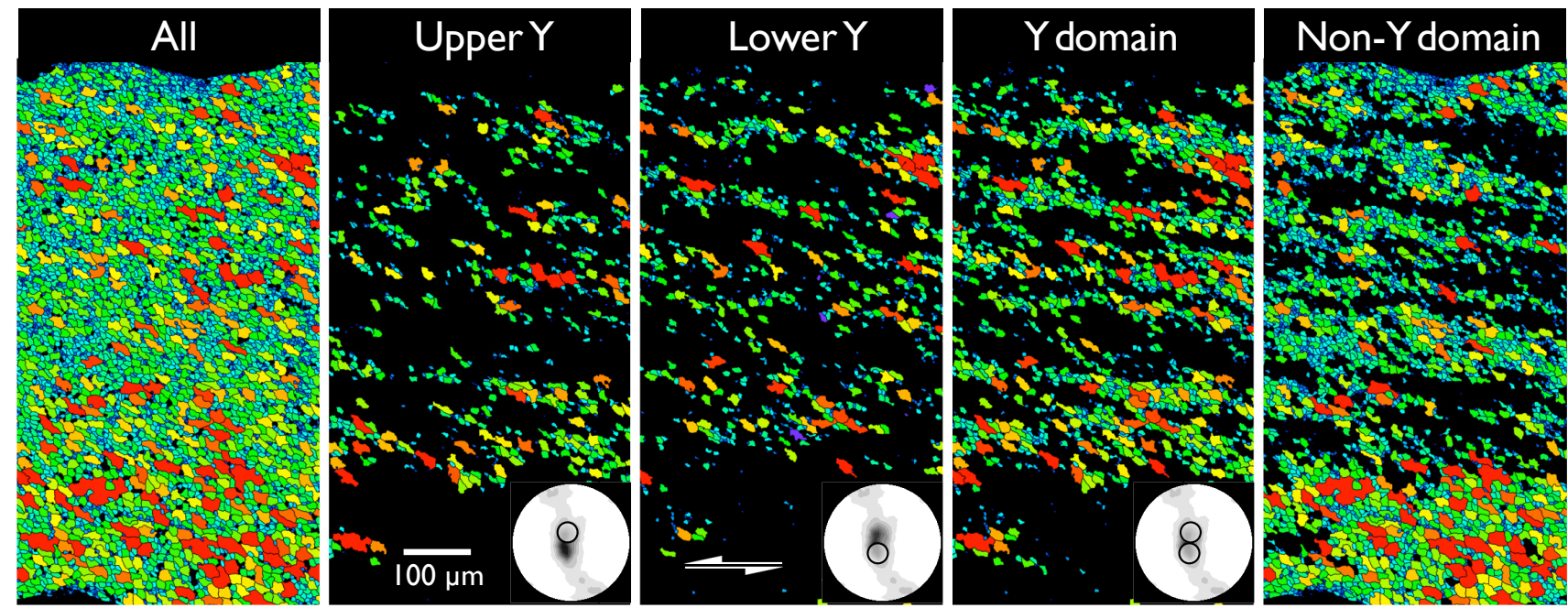

\section{2-D}
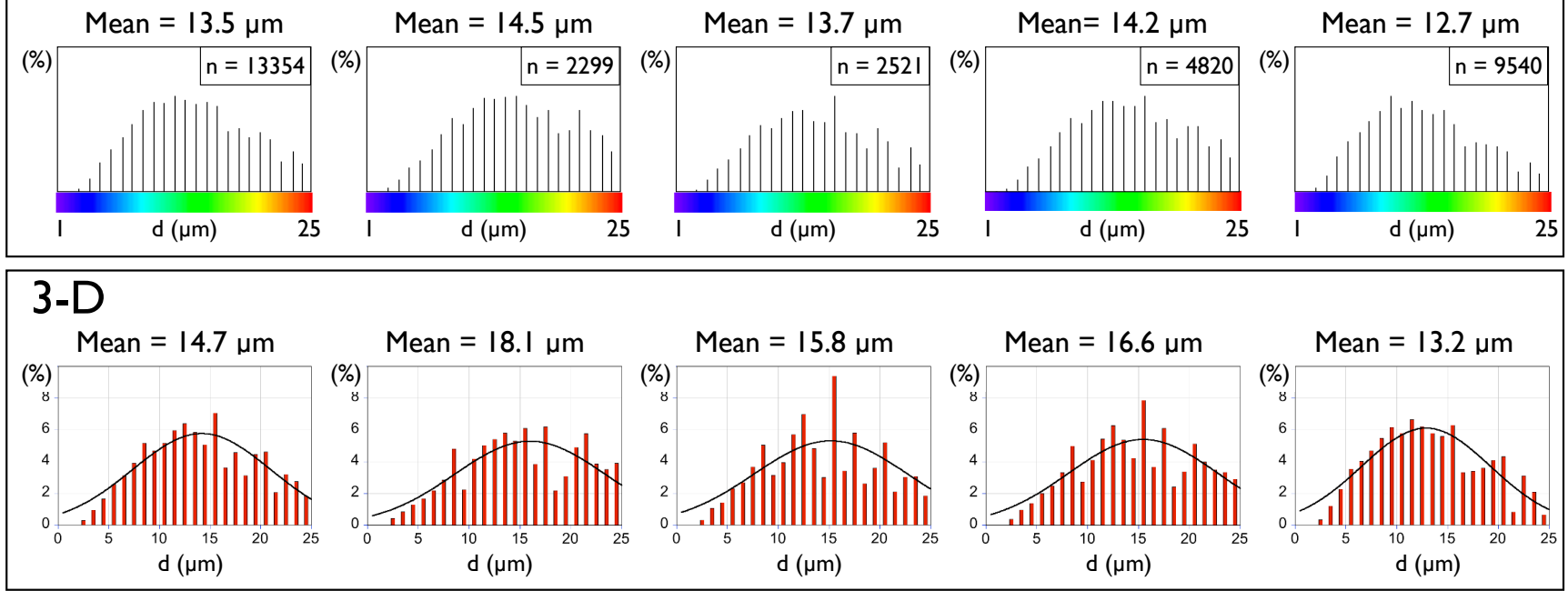

Figure 8. Grain size in texture domain. Grain size maps of sample w935 (regime 3) are shown. From left to right: all grains, grains in the upper and lower Y subdomains (as indicated by the inset pole figures), in the whole $\mathrm{Y}$ domain, and for $c$-axis orientations outside the $\mathrm{Y}$ domain. All domain maps are derived for $c$-axis orientations within a $30^{\circ}$ cone $\left(15^{\circ}\right.$ opening angle) with respect to the central orientation. Domain maps are derived for $c$-axis orientations within a $30^{\circ}$ cone $\left(15^{\circ}\right.$ opening angle $)$ with respect to the central orientation. Scale bar and shear sense apply to all. Below the maps are area-weighted distributions of 2-D grain size (gray value histogram of grain size map; see Heilbronner and Barrett, 2014, 14 chap. 12) with the mean indicated above, and histograms of StripStar-calculated volume fractions of the 3-D grain size with the mean (= mode) of the Gauss fit. Note that the grain size in the $\mathrm{Y}$ domains is larger than in the non-Y domain, while the difference between the upper and lower Y subdomain is not significant.

ues for regime 3 are confirmed by the present study, which yields a value of $14.7 \mu \mathrm{m}$ (w935). As the regime 1 and 2 grain sizes are too small to be properly resolved by the light optical input of the CIP method, it is not surprising that the grain sizes derived from EBSD input are smaller with values of $5.0 \mu \mathrm{m}$ (w940) and $6.3 \mu \mathrm{m}$ (w946) (Table 2).

\subsection{Factors influencing the recrystallized grain size}

\subsubsection{Dependence of grain size on stress}

When Hirth and Tullis (1992) introduced the dislocation creep regimes, the regimes 1,2 , and 3 were defined by the microstructure. At the same time, the boundaries between the regimes were observed to be constant stress boundaries, placing regime 1 above a differential stress, $\Delta \sigma$, of $400 \mathrm{MPa}$, regime 2 at approximately $300 \mathrm{MPa}$, and regime 3 at or below $200 \mathrm{MPa}$. For shear experiments, these values translate to shear stresses, $\tau$, of 200,150 , and $100 \mathrm{MPa}$, respec- 


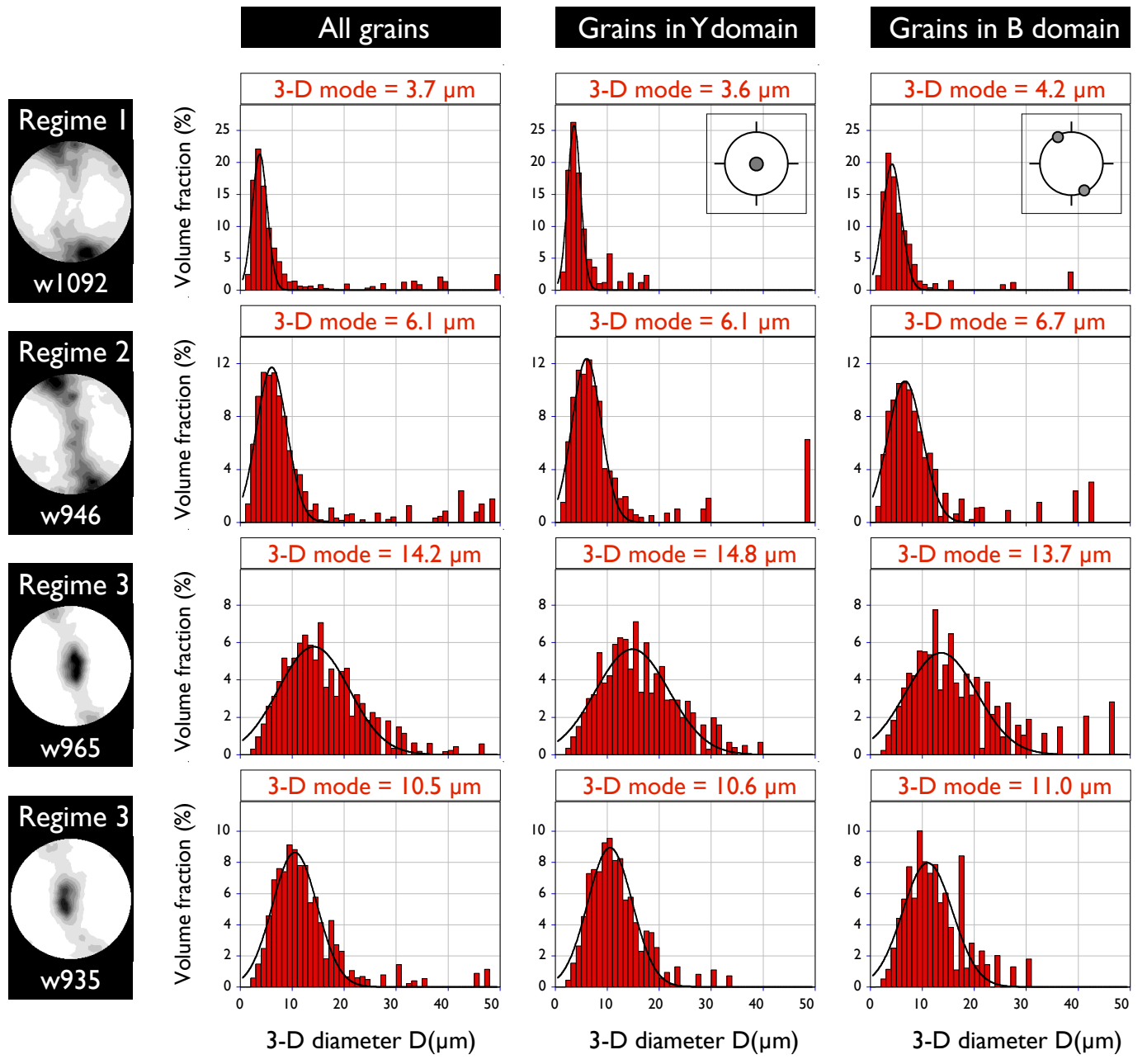

Figure 9. Recrystallized grain size as a function of texture. Grain size distributions of recrystallized grains for four samples of regimes 1 , 2 , and 3 of dislocation creep, arranged in four rows with $c$-axis pole figure on the left. From left to right: grain size distributions of all recrystallized grains and those within the $\mathrm{Y}$ and $\mathrm{B}$ domains. $D=$ diameter of volume equivalent sphere. The mode of $v(D)$ is obtained by a Gauss fit to the distribution. Inset (upper right) schematically shows the location of the corresponding B and Y maxima on the pole figure.

tively, which are values that were realized, e.g., in the studies of Heilbronner and Tullis $(2002,2006)$. The stress-strain curves of the samples analyzed here (Fig. 1) also confirm this trend to close approximation. Only the low-strain sample of regime 2 (w1086) deforms at a higher shear stress than the low-strain sample of regime 1 (w940), raising the question of whether w1086 should actually be counted as regime 1 . When plotting the recrystallized grain sizes against differential stress, the high-strain samples follow a clear trend (Fig. 10), however, at higher stress levels than predicted by the quartz piezometer of Stipp and Tullis (2003). Including the low-strain samples does not significantly alter the picture. On a line fit, higher stresses or higher grain sizes are predicted compared to the piezometer. In the case in which the grain size is given as the 3-D mode (red curves in Fig. 10), this is to be expected because the 3-D mode is always larger than the 2-D RMS for which the piezometer was calculated.
However, the curves fitted to the 2-D RMS values (green curves in Fig. 10) also yield higher stresses or grain sizes.

Where does this discrepancy come from? A number of explanations are possible. Heilbronner and Tullis (2006) attributed the higher stresses to the use of a solid confining medium as compared to the molten salt assembly that had been used for the piezometer experiments. If the correction proposed by Holyoke and Kronenberg (2010) were to be used, the curve fit would shift to lower stresses but still remain significantly above the piezometer. However, this correction was not used for the recalculation of the stress-strain curves because without it, the new rigS software achieved correct stresses as calibrated against the quartz-coesite transition (Richter et al., 2016).

Comparing general shear experiments to axial shortening ones requires a conversion of shear stress to differential stress. Lower differential stresses could result if, instead of 


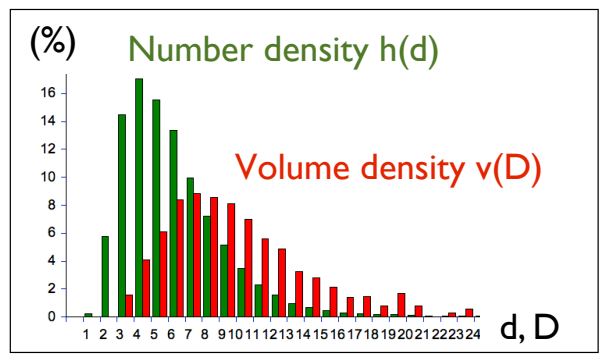

$$
\begin{aligned}
& \text { - RMS of } h(d) \\
& - \text { Mode of } v(D)
\end{aligned}
$$$$
d=\text { Diameter of 2-D circles }
$$$$
\mathrm{D}=\text { Diameter of 3-D spheres }
$$

Piezometer of Stipp and Tullis (2003):

$$
\begin{aligned}
-\mathrm{d}(\mu \mathrm{m}) & =363 \mathrm{I} \cdot \Delta \sigma^{(-1.26)} & & \text { Regime 2 \& } 3 \\
\mathrm{~d}(\mu \mathrm{m}) & =78 \cdot \Delta \sigma^{(-0.6 \mathrm{I})} & & \text { Regime I }
\end{aligned}
$$

\section{(a) High-strain samples}

\section{(b) Seven samples}

\section{(c) Texture domains}
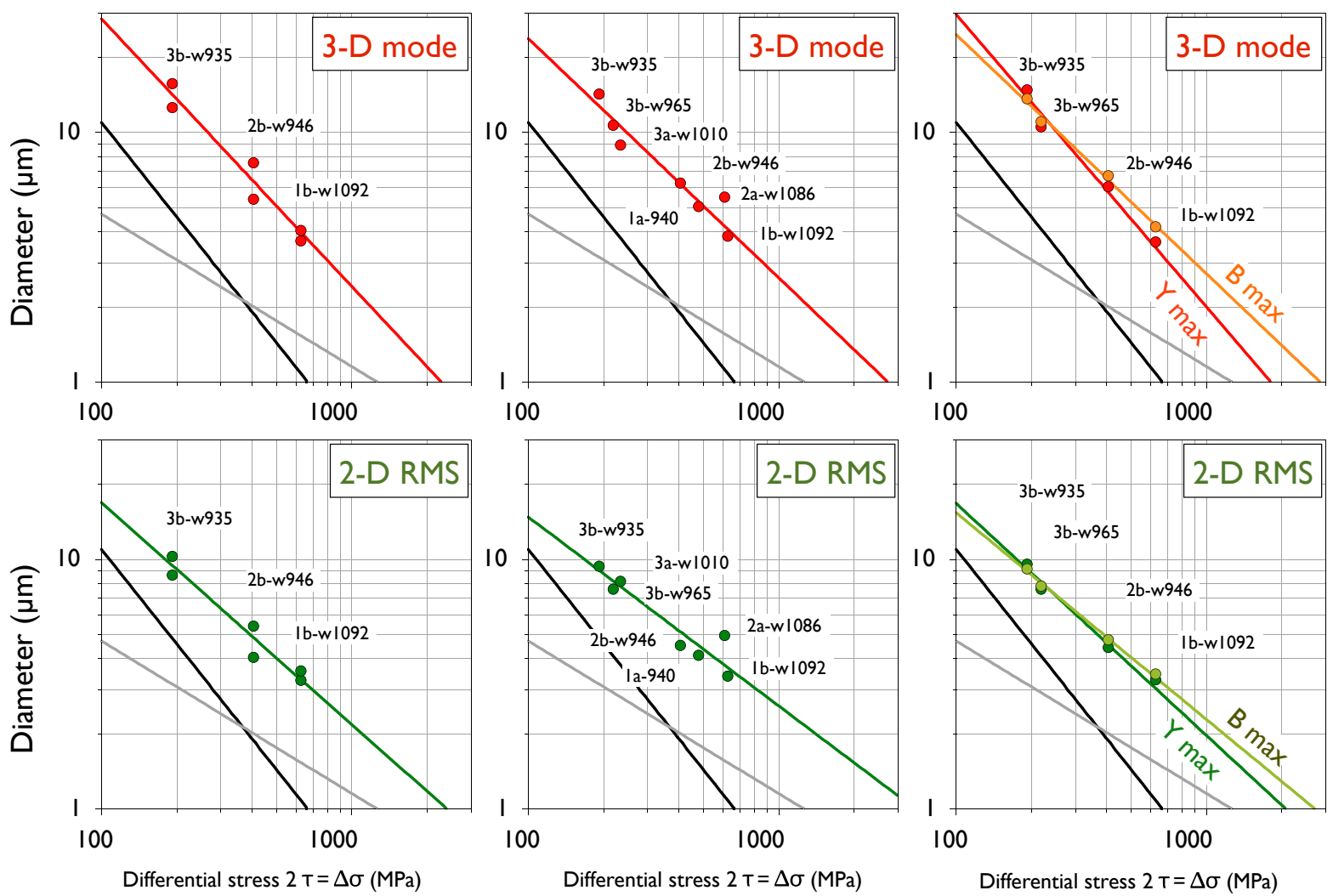

Figure 10. Recrystallized grain size as a function of flow stress. Two measures of average grain size are plotted against differential stress, $\Delta \sigma$ (with $\Delta \sigma=2 \tau$; see Table 1). Top row: mode of $v(D)$ where $D=$ diameter of volume equivalent sphere and $v=$ volume weighted frequency distribution ( $=3$-D mode). Bottom row: root mean square of $h(d)$ where $d=$ diameter of area equivalent circle and $h=$ frequency distribution as used for the piezometer relation by Stipp and Tullis (2003) (=2-D RMS). Separate plots are shown (a) for three high-strain experiments $(5.8<\gamma<7.1)$ with the higher value deriving from the low-gKAM region and the lower value from the high-gKAM region (see Fig. 6) (b) for the bulk measurement of each of the seven experiments (see Table 1) and (c) for the grain sizes of the Y and B domains (see Table 2). The piezometer curves for regimes 2 and 3 (black) and for regime 1 (gray) of Stipp and Tullis (2003) are always included for reference.

the Mohr circle construction, $\Delta \sigma=2 \tau$, smaller conversion factors could be used. For torsion experiments, $\Delta \sigma=\sqrt{3} \tau$ (Paterson and Olgaard, 2000), and if the stress exponent $n$ is considered, using $\tau=3^{k} \Delta \sigma$ (where $k=-\frac{1+1 / n}{2}$ ), a range of values from $\Delta \sigma=3 \tau$ (for $n=1$ ) and $\Delta \sigma=\sqrt{3} \tau$ (for $n \gg 1$ ) is possible (Ranalli, 1987; Schmid et al., 1987). In other words, none of these theoretically possible conversion fac- tors produces an acceptable overlap of the curve fits and the piezometer. This raises the question of whether the regimes and regime boundaries of the general shear experiments correspond one-to-one to those of the axial shortening experiments. However, because more data would be needed to explore the implications of this observation, this line of argument is not pursued further here. 
The piezometer experiments of Stipp and Tullis (2003) were conducted to relatively low finite strains and were stopped long before $100 \%$ volume fractions of recrystallization were achieved. However, including low-strain experiments (Fig. 10) shows that the mismatch cannot be due to different amounts of recrystallization. An interesting question is whether the piezometer relation is restricted to coaxial progressive deformation and does not apply to non-coaxial progressive deformation; however, the answer to this question is again outside the scope and focus of this study.

In their study on texture evolution in regime 3 dislocation creep, Heilbronner and Tullis (2006) found that the recrystallized grains in the prism domain (here the $\mathrm{Y}$ domain) are larger than the grains of other domains. Considering inverse grain boundary density (Fig. 12c in Heilbronner and Tullis, 2006), the size ratio between the recrystallized grains in the $\mathrm{Y}$ domains compared to the average reaches a value of 1.4 at high shear strains. The same figure also predicts that the ratio for the so-called basal domain (here the $\mathrm{B}$ domain) should attain a value of 1.0 or even $<1.0$. To check these claims for regime 3 , and to check whether they also apply to regime 1 and 2 samples, maps of texture domains were prepared and the grain size of the $\mathrm{Y}$ and $\mathrm{B}$ domain determined (Figs. 8 and 9). For regime 3, it is found that the 3-D mode of the recrystallized grains of the bulk is $14.7 \mu \mathrm{m}$, that of the $\mathrm{Y}$ domain $16.6 \mu \mathrm{m}$, and that of the non-Y domain $13.2 \mu \mathrm{m}$ (Fig. 8). However, the ratio of the recrystallized grain size in the Y domain to the average is only 1.1 (w935) and 1.01 (w965), while the ratio of the B domains to the average is 1.0 (w935) and 1.05 (w965; Fig. 9). In other words, the expectation that $\mathrm{Y}$ domains in regime 3 have a larger recrystallized grain size and B domains an average or smaller grain sizes is confirmed, if not to the full extent of the predictions made in 2006. Note that for the study presented in the paper by Heilbronner and Tullis (2006), the available database was not sufficiently large and the method of size estimation via grain boundary density not well-enough calibrated to allow for a quantitative prediction. Nevertheless, it could be documented that the relative size of the recrystallized grains of the $\mathrm{Y}, \mathrm{B}$, and other domains evolve continuously with increasing shear strain.

In this study another interesting point emerges: the ratio between the recrystallized grain sizes in the different domains depends on the regime and may change depending on the stress level. For regimes 1 and 2, the size ratios of the recrystallized grains in the $\mathrm{Y}$ and $\mathrm{B}$ domains with respect to the average can also be extracted from Fig. 9. Proceeding from the strongest to the weakest sample (regime $1 \mathrm{w} 1092$, regime $2 \mathrm{w} 946$, regime $3 \mathrm{w} 965$ and w935), the size ratio for the $\mathrm{Y}$ domain is $0.95,1.0,1.0,1.1$, and for the $\mathrm{B}$ domain 1.1, $1.1,1.05,0.97$, respectively. The resulting stress dependence of the recrystallized grain size in the $\mathrm{Y}$ and $\mathrm{B}$ domains is shown in Fig. 10. In view of the subtle difference between the two and the possible errors associated with the stress deter- minations, it is not clear if this result, however provocative, is significant at all, but it certainly merits additional study.

\subsubsection{Dependence of grain size on the CPO (domain scale)}

The misorientation density as measured by the gKAM can be interpreted as an indicator of deformation intensity; in the case of continuous subgrain rotation recrystallization, more highly deformed, recrystallized grains have a higher density of low-angle boundaries and/or low-angle boundaries with higher misorientation angles. Thus, highly deformed grains have high gKAM values. Comparison of the grain size maps (Fig. 4) with the maps of the gKAM (Fig. 6) shows that regions with high overall gKAM values are also regions of overall smaller grain size. Gradients of grain size and gKAM may not always be as well developed as in the samples shown in Fig. 6. The absence of such gradients is probably the result of homogeneous deformation of the sample (across the entire width of the shear zone), whereas gradients point to strain concentrations. For regions with constant gKAM, however, the size ratios between texture domains persist, as can be seen by comparing the map for the $\mathrm{Y}$ domain and that for the non-Y domain in Fig. 8. They both show an overall size increase from top to bottom, but at every level on that traverse, the $\mathrm{Y}$ domain shows larger grain sizes than the non-Y domain. For sample w935 (shown in Fig. 8), the ratio between the $\mathrm{Y}$ and non-Y domain is 1.2. In other words, while the overall recrystallized grain size is inversely correlated to the level of the gKAM, the gKAM itself does not give rise to the grain size difference between different texture domains.

\subsubsection{Dependence of grain size on misorietation density (grain scale)}

Regardless of the absolute stress levels of the experiments discussed here, a relation of misorientation density and recrystallized grain size can be documented. The stress-grain size relation (Fig. 10) is calculated using only the high-strain and more or less fully recrystallized samples. In view of the grain size gradients across these samples (Fig. 3) and the relation of these on the gKAM (Fig. 6), a low- and a highgKAM site are used in each case. In all cases, lower gKAM values coincide with larger recrystallized grain size. While each pair is plotted for the shear stress determined for the sample, it is quite possible that the grain size gradient in fact indicates a strain rate gradient caused by the localization of the deformation into a narrow active zone and which would, according to the piezometric interpretation, result in a stress increase within the shear zone. Progressive thinning of the samples has been used as an explanation for the apparent strain hardening at the end of long shear experiments (Heilbronner and Tullis, 2002). Raised stress levels may also occur at the grain scale, both in the function of the crystallographic orientation of the grain with respect to the principal 


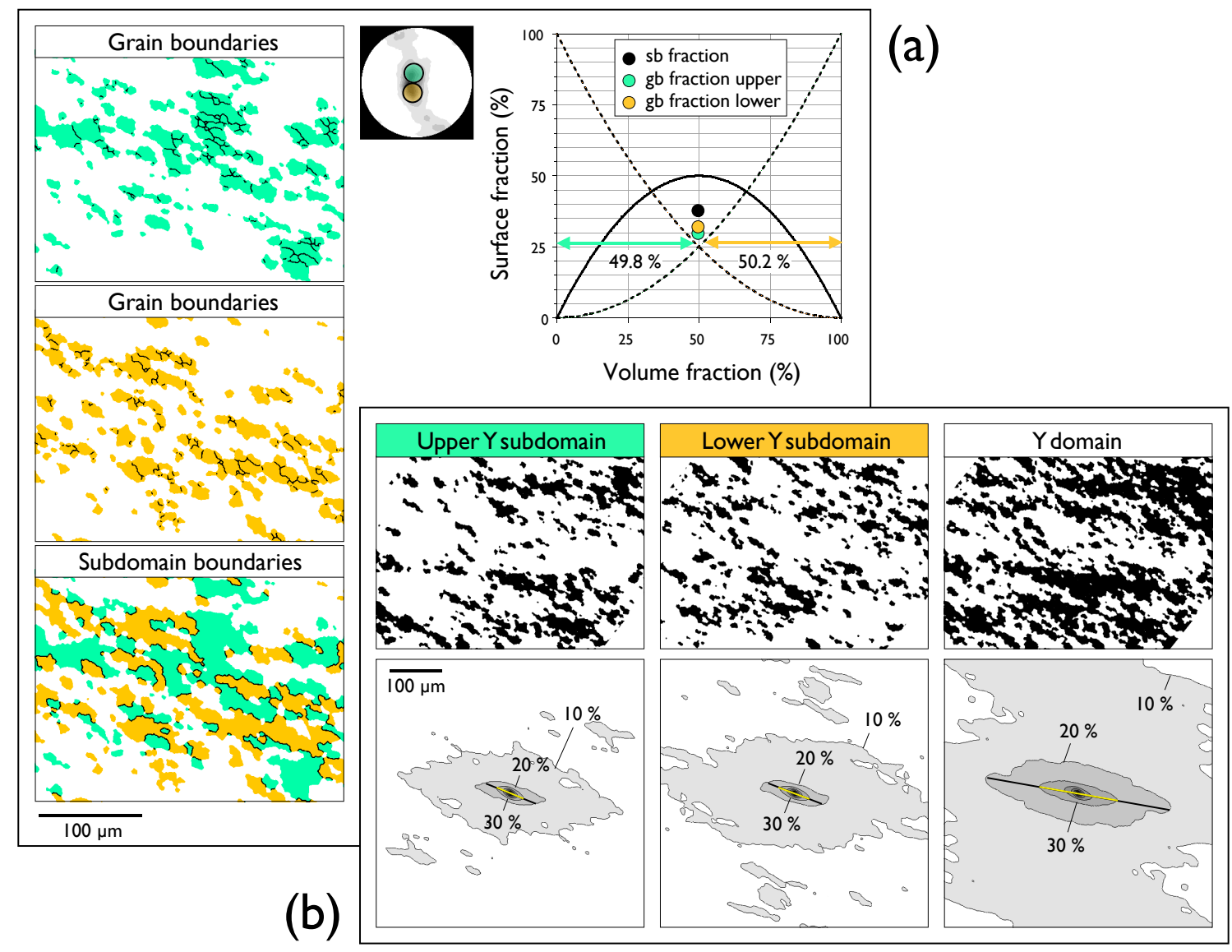

Figure 11. Spatial distribution and cluster size of Y subdomains. Results are shown for sample w935 of regime 3. (a) Spatial analysis to determine if the spatial distribution of subdomains is random, clustered, or ordered (anti-clustered). Maps of subdomains with grain and subdomain boundaries are shown: "upper" (green) and "lower" (yellow) refer to the upper and lower maximum of the pole figure shown on the right, and the scale bar below applies to all. Upper right: plot showing fraction of subdomain boundary (sb) and grain boundary (gb) vs. volume fraction of the subdomains (upper : lower $=49.8: 50.2 \%$ ). Solid and stippled lines are the expected surface fractions for the random case. Fraction sb below and fractions gb above the expected values indicate significant clustering. (b) Autocorrelation analysis of domain and subdomains: thresholded maps with autocorrelation function (ACF) below. Long and short diameters of contours in ACF reflect size and shape of clusters in domain and subdomain maps. Scale bar applies both to ACFs and maps. Three contour levels are indicated as a percentage of ACFmax. Superposed yellow lines indicate long diameter and orientation of $30 \%$ contour; black lines are extrapolations to $20 \%$ contour (see text).

kinematic framework and at grain-to-grain contacts as long as recrystallization is incomplete. It is therefore possible that shear stresses determined for bulk samples are different from the stress "felt" locally by the actively deforming material.

\subsection{Kinematic analysis of texture domains}

It is interesting to note that the $\mathrm{Y}$ domain and the $\mathrm{B}$ domain are arranged as layers with clusters of grains belonging to one or the other submaximum in the pole figure (see Fig. 5). Testing the neighborhood relations between grains of the submaxima (using the spatial analysis described in Chap. 18 of Heilbronner and Barrett, 2014) revealed that they are not randomly arranged within the layers but rather strongly clustered (Fig. 11a). Pole figures with double Y maxima have been shown repeatedly in a number of publications; however, no particular attention has been given to them (see e.g., Mancktelow, 1987; Stipp et al., 2002; Pennacchioni et al., 2010; Law, 2014). In order to determine the shape of texture domains, the autocorrelation function (ACF) is used. Superposed lines on the ACFs (Fig. 11b) represent the orientations of the $30 \%$ contours. Their trend with respect to the shear direction is 22 and $21^{\circ}$ for the upper and lower subdomain, respectively, and $10^{\circ}$ for the combined $\mathrm{Y}$ domain. Obviously, the shallow trend of the Y domain is the result of an imbricate arrangement of the more steeply inclined subdomains. On account of the shallow trend of the Y domain, Heilbronner and Tullis (2006) argue that while all domains deform as particles of higher viscosity in a low viscosity matrix (using the approach by Gay, 1968), the prism domain is the one 


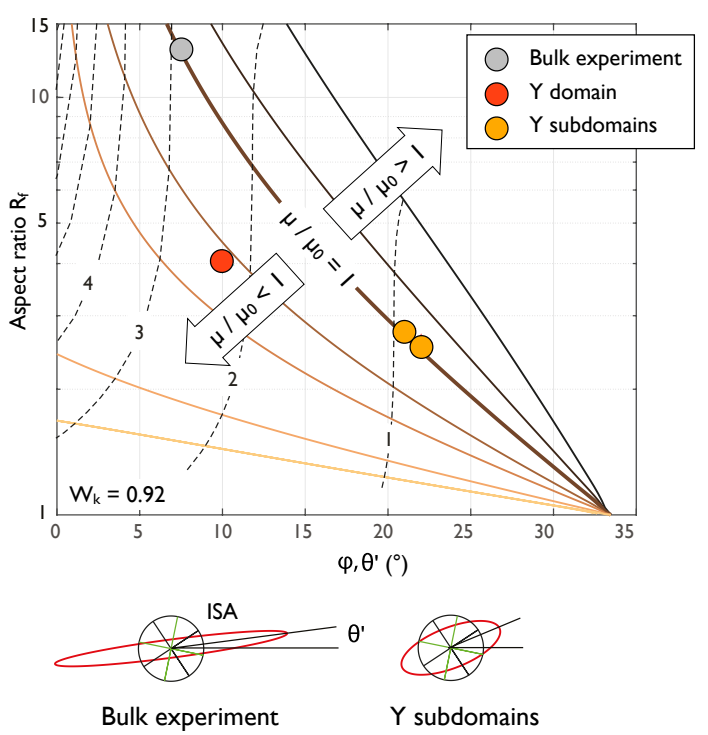

Figure 12. Relation of quartz domains and bulk sample strain. $R_{\mathrm{f}}-\phi$ type plot calculated for general shear. Contours (solid lines) are for viscosity ratios $\kappa=\mu / \mu_{0}$ of $0.01,0.5,1,2,3,6$, and 9 calculated for the linear viscous case. Dashed lines represent constant shear strain. For w935, the ratio of principal stretches $R_{\mathrm{f}}=12.9$; the trend of the major axis of the strain ellipse $\Theta^{\prime}=7.5^{\circ}$; the kinematic vorticity number $\mathrm{W}_{k}=0.92$. Aspect ratios of the $\mathrm{Y}$ subdomains are 2.5 and 2.7; the corresponding trend of the long axes $(\phi)$ is 22 and $21^{\circ}$. Strain ellipses for bulk sample and Y subdomains are shown below.

with the lowest viscosity ratio $(\kappa)$ of them all, having $\kappa \approx 2$ (where $\kappa=\mu / \mu_{0}, \mu$ is the viscosity of the domain and $\mu_{0}$ the viscosity of the matrix) and making it the "softest" among all domains.

In this contribution, however, we prefer not to pursue the approach by Gay (1968) because of the shortcomings and errors associated with it. Instead we first calculate an $R_{\mathrm{f}}-\phi$ diagram for various viscosity ratios (Fig. 12) based on the procedure described by Mancktelow (2011) using the approach of Bilby and Kolbuszewski (1977). Next, we calculate the finite strain of the bulk sample assuming homogeneous, continuous general shear according to Fossen and Tikoff (1993). Plotting the ACF-derived aspect ratio and orientation $\left(R_{\mathrm{f}}-\phi\right.$ coordinates) of the domain and subdomain clusters into the $R_{\mathrm{f}}-\phi$ diagram (Fig. 12) reveals that the subdomains plot on the equi-viscous curve $(\kappa=1)$, as does the bulk sample by default, while the full domain plots on a curve for a viscosity ratio $>1$. Note that the true shear strains of the subdomains $\left(\gamma_{\text {true }} \sim 1\right)$ remain significantly lower than the total true shear strain for the bulk sample w935 with $\gamma_{\text {true }} \sim 3$. This also implies that subdomains deform at a lower rate than the bulk sample, while they are still equi-viscous with respect to their neighboring domains. Such a situation, however, requires an additional deformation mechanism operative throughout the entire sample to obtain total observed strain. One possible and likely candidate is grain boundary sliding, which has been suggested for regime 1 experiments (Stipp and Kunze, 2008; Kidder et al., 2016); however, that it could also contribute to bulk strain in regimes 2 and 3 was not suspected, and additional evidence is given in the companion paper (Kilian and Heilbronner, 2017).

Another interesting point to note is the ratio of the apparent shear strain, usually reported as $\gamma$ in deformation experiments (see Fig. 1), to the effective shear strain $\gamma_{\text {eff }}$ of $\sim 1.6$ (see Table 1). When comparing experimental microstructures to natural ones, it might be more reasonable to use the true shear strain for comparison with the shear strain measured in the field. A number of alternative measures derived from the initial and the final thickness of the shear zone (Gleason et al., 1993) or methods for the incremental calculation of shear strain (Richter et al., 2016) have been proposed, always assuming progressive simple shear. However, the true shear strain or the effective shear strain (e.g., Fossen and Tikoff, 1993) returns values that are smaller and may be closer to values that are relevant in nature. Using the apparent shear strain (the highest possible) may be part of the reason why in nature steady-state microstructures and textures appear to be established at much lower strains than in experiments (see discussion by Pennacchioni et al., 2010). It also means that care should be taken when using the relation between volume fractions of recrystallized grains and the apparent shear strain as determined from general shear experiments to estimate the shear strain in nature (Rahl and Skemer, 2016).

To assess the size of the subdomains, we consider the ACF again. The long diameters of the $30 \%$ contours (typically used for size estimates; see Chap. 20 in Heilbronner and Barrett, 2014) are 59, 64, and $153 \mu \mathrm{m}$, and those of the $20 \%$ contours 103,111, and $356 \mu \mathrm{m}$ again for the upper and lower subdomains and the combined $\mathrm{Y}$ domain, respectively. Both measures indicate that the cluster size of the combined domain is approximately 3 times the cluster size of the subdomains. This led Heilbronner and Tullis (2006) to the conclusion that the prism domains could not represent original BHQ grains (of prism orientation) but must have grown by coalescence of preferentially replacing "harder" basal and $\sigma_{1}$ grains by "softer" prism grains through preferred recrystallization and coalescence. Considering now that the subdomain clusters have about the same diameters as the original BHQ grains and that their orientation is compatible with strain, another interpretation is possible: subdomain clusters could indeed be the strained "ghosts" of the original BHQ grains with strain by a crystal plastic mechanism bringing their $c$ axes close to a common $Y$ direction but never into parallelism.

\section{Summary and conclusions}

A microstructure and texture analysis of seven samples of Black Hills Quartzite, deformed experimentally in the dislo- 
cation creep regimes 1,2 , and 3 , was carried out with the aim of comparing previously published data obtained by the CIP method to a renewed analysis making use of the higher resolution (both spatially and in terms of crystallographic orientation) of EBSD.

1. In order to best reproduce the visual identification of grains, segmentations have to be performed using a $c$ axis misorientation angle of $3^{\circ}$, which is approximately equivalent to a full misorientation angle of $5^{\circ}$.

2. Comparison of CIP and EBSD method: For grain sizes $>10 \mu \mathrm{m}$, orientation maps and derived grain sizes coincide. For smaller grain sizes, however, optical resolution is insufficient, and EBSD should be used.

3. The recrystallized grain size depends

- on the level of the shear stress supported by the sample during the experiment;

- locally, on the CPO of a given texture domain; and

- locally, on the deformation intensity as measured by the misorientation density (gKAM).

Thus, the averaged recrystallized grain size does not depend on the total strain achieved by the sample or the volume fraction of recrystallization, but in detail on the local strain in the sample.

4. The Y domain (identified previously as the prism domain) is composed of two subdomains, and the same is true of the B domain (basal domain).

5. The size of the Y subdomains corresponds to the original grain size of BHQ. The shape and long axis trend suggest that they deform equi-viscous to the bulk experiment but potentially to a lower shear strain than the bulk experiment, calling for an additional deformation mechanism other than dislocation creep.

6. The recrystallized grain sizes of the $\mathrm{Y}$ and $\mathrm{B}$ domains appear to have different stress dependences; i.e., under the assumption of iso-stress conditions, the grain size of $\mathrm{Y}$ and $\mathrm{B}$ domains defines different piezometric relationships.

7. The stress dependence of the recrystallized grain size of the shear experiments analyzed here predicts higher stress for a given grain size or larger grain size for a given flow stress than the piezometer of Stipp and Tullis (2003).
Future work is suggested to examine whether the discrepancy between the grain sizes obtained here and the published piezometer are only due to discrepancies between the stress calculations for solid-medium confining pressure as opposed to the molten salt assembly, as was used for the piezometer experiments. If so, this would suggest that the stresses reported in the literature for experiments carried out with solidmedium confining pressures are too high by a factor of 2 or more (a rather alarming situation). Discrepancies could also arise from the state of stress experienced by the recrystallized grains, which may deviate considerably from the stress supported by the bulk sample. Alternatively, it may indeed show that coaxial and non-coaxial progressive deformation produce different recrystallized grain sizes.

Data availability. The data sets used in this paper are not yet publicly available. Please contact the lead author for access. 


\section{Appendix A: Segmentation and grain size analysis}

\section{A1 Procedure to obtain CIP boundaries}

Segmentation is carried out using Image SXM and the Lazy Grain Boundaries (LGB) macro (Heilbronner and Barrett, 2014). The input consists of eight $c$-axis misorientation images (MOI) calculated with respect to four external reference directions and four internal reference directions corresponding to the four most prominent maxima in the pole figure. The MOIs are combined to a stack and resized by a factor of 2 (or 4) using nearest neighbor interpolation (NN) to preserve the calculated pixel values and to retain sharp boundaries. The sequence of steps necessary to complete segmentation is listed in Appendix Table A2 in the form of LGB keystrokes. Typically the contrast of each slice is optimized by histogram equalization $e$. Edge detection (Sobel operator) is preformed $o$, and the eight gradient images are OR added $z$, keeping the maximum value (of the eight gradient images) at each pixel. The resulting image is thresholded at a gray value between 25 and 50 according to visual impression; these are values that correspond to approximately $1.2-2.5^{\circ}$ ( $c$-axis misorientation). One or two rounds of thickening $t$, skeletonizing $j$ and pruning $i$ are applied to obtain grain boundaries that are 1 pixel wide. Depending on the noise caused by mis-indexing, additional median filtering needs to be applied during the segmentation to the slices of the stack $u$ or to the combined image $m$. To obtain the final grain map, the grain boundaries are thickened to a width of 2 pixels and the grain boundary map (black lines on white background) is inverted. At this point, the grain map (black segments) consists of all possible "grains", including those that consist of a hole, dirt, or a different mineral phase. This is so because grain boundary detection not only detects high gradients between indexed pixels of different $c$-axis orientation, but also between indexed and non-indexed pixels. Such "grains" of nonindexed pixels are excluded from future analyses. As will be shown in the next section, this is accomplished through "redirect sampling" and by analyzing the grain map together with the mask image (i.e., the map of the indexed pixels).

\section{A2 Procedure to obtain EBSD boundaries}

Segmentation of grains from EBSD data can be accomplished based on a misorientation angle threshold assuming that grains are objects enclosed by boundaries that fulfill the segmentation criterion at every point along the boundary. Here, a minimum angle of misorientation of $10^{\circ}$ is chosen. In MTEX, the measurement points do not have to be located on a regular grid, although they usually are, nor do they have to be in direct contact with one another. It is possible to reconstruct grains that are dissected by arrays of non-indexed pixels (e.g., scratches) as long as the misorientation angle between the disconnected pieces is below a given threshold and spatial conditions are fulfilled (Bachmann et al., 2011). It is also possible to attribute fractions of non-indexed pixels to the closest grain, i.e., to an indexed area, based on certain textural or spatial criteria. This procedure, in the following called grain completion, generates grain boundaries that outline "completed" grains, i.e., grains consisting of indexed pixels and "incorporated" areas. The degree of grain completion has to be adapted to the individual image quality; therefore, the process of grain completion needs to be supervised. The most conservative approach is to use no grain completion at all; at the other end of the spectrum is the total completion, which leaves no pixel unassigned. The resulting grain boundaries for segmentation based on total completion and grain boundaries obtained without grain completion are shown in Appendix Fig. A1. In contrast to the grain boundary bitmaps obtained by image analysis such as the CIP method, EBSD grain boundaries have zero thickness and in the case of grain completion, the grain sizes need not be integer multiples of the step size. 
Table A1. EBSD data acquisition.

\begin{tabular}{|c|c|c|c|c|c|c|c|c|c|c|c|c|c|c|}
\hline 1 & 2 & 3 & 4 & 5 & 6 & 7 & 8 & 9 & 10 & 11 & 12 & 13 & 14 & 15 \\
\hline Sample & $\begin{array}{r}\text { Voltage } \\
\mathrm{kV}\end{array}$ & $\begin{array}{r}\text { Probe } \\
\mathrm{nA}\end{array}$ & $\begin{array}{r}\text { Pressure } \\
\mathrm{Pa}\end{array}$ & $\begin{array}{r}\text { Aperture } \\
\mu \mathrm{m}\end{array}$ & $\begin{array}{l}\text { WD } \\
\text { mm }\end{array}$ & Magn. & $\begin{array}{r}\text { Speed } \\
\mathrm{Hz}\end{array}$ & $\begin{array}{l}\text { Time } \\
\mathrm{h}: \mathrm{m}\end{array}$ & Bands & Mean MAD & Hough res. & Binning & $\begin{array}{r}\text { Step size } \\
\mu \mathrm{m}\end{array}$ & $\begin{array}{r}\text { Map size } \\
\mu \mathrm{m}\end{array}$ \\
\hline \multicolumn{15}{|c|}{ Undeformed material } \\
\hline $\mathrm{BHQ}^{*}$ & 20 & 5 & 35 & 120 & 9.5 & $200 \times$ & 41 & $9: 19$ & $48 / 9$ & 0.58 & 120 & $4 \times 4$ & 1 & $1388 \times 980$ \\
\hline \multicolumn{15}{|c|}{ Unscanned sites of experiments } \\
\hline w940 & 20 & NA & 2 & 120 & 14.5 & $250 \times$ & 23 & $17: 50$ & $75 / 9$ & 0.89 & 70 & $2 \times 2$ & 0.5 & $500 \times 725$ \\
\hline w1092** & 20 & NA & 28 & 120 & 14.5 & $250 \times$ & 23 & $18: 45$ & $75 / 10$ & 0.9 & 110 & $2 \times 2$ & 0.5 & $550 \times 700$ \\
\hline w1092-s30 & 20 & NA & NA & NA & 14.7 & NA & 11 & $10: 48$ & $75 / 9$ & 0.81 & 70 & $2 \times 2$ & 0.5 & $241.5 \times 452$ \\
\hline w1086 & 20 & 3 & 20 & 120 & 14.6 & $150 \times$ & 23 & $05: 54$ & $75 / 9$ & 0.9 & 70 & $2 \times 2$ & 0.5 & $600 \times 200$ \\
\hline w946** & 20 & NA & 28 & 120 & 13.5 & $300 \times$ & 23 & $18: 16$ & $75 / 10$ & 0.62 & 110 & $2 \times 2$ & 0.5 & $750 \times 485$ \\
\hline w1010-s34 & 20 & 9 & 25 & 120 & 14.3 & $200 \times$ & 40 & 03:02 & $75 / 9$ & 0.78 & 70 & $4 \times 4$ & 1 & $430 \times 980$ \\
\hline w1010-s36 & 20 & 9 & 25 & 120 & 14.3 & $200 \times$ & 11 & $02: 51$ & $75 / 9$ & 0.84 & 70 & $2 \times 2$ & 1 & $500 \times 830$ \\
\hline w935** & 20 & NA & 28 & 120 & 13.4 & $200 \times$ & 23 & $15: 58$ & $75 / 10$ & 0.57 & 110 & $2 \times 2$ & 1 & $1277 \times 1027$ \\
\hline w965-s40 & 20 & 6 & 25 & 120 & 15 & $150 \times$ & 40 & $14: 28$ & $75 / 9$ & 0.82 & 70 & $4 \times 4$ & 1 & $840 \times 700$ \\
\hline w965-s45 & 20 & 3 & 20 & 120 & 14.8 & $250 \times$ & 23 & $14: 00$ & $75 / 10$ & 0.75 & 70 & $2 \times 2$ & 0.25 & $180 \times 400$ \\
\hline
\end{tabular}

Table A2. Image processing and segmentation.

\begin{tabular}{|c|c|c|c|c|c|c|c|c|c|c|c|}
\hline 1 & 2 & 3 & 4 & 5 & 6 & 7 & 8 & 9 & 10 & 12 & 12 \\
\hline Regime & Map & Source & $\begin{array}{r}\text { Crop size } \\
\text { px }\end{array}$ & $\begin{array}{r}\text { Hit rate raw } \\
\%\end{array}$ & $\begin{array}{r}\text { Hit rate deN } \\
\%\end{array}$ & $\begin{array}{r}\text { Step size } \\
\mu \mathrm{m}\end{array}$ & Images & Magn. & $\begin{array}{r}\text { Pixel size } \\
\mu \mathrm{m}\end{array}$ & Procedure & $\begin{array}{r}\text { Def. } \\
{\left[{ }^{\circ}\right]}\end{array}$ \\
\hline & BHQ & EBSD & $1388 \times 980$ & 91.4 & 94.5 & 1 & 8 misors & 1 & 1 & LGB interactive & NA \\
\hline & bhq $2.5 \times$ & CIP & $1388 \times 1040$ & - & - & 2.4 & nopol & 1 & 2.4 & visual boundaries & NA \\
\hline $1 \mathrm{a}$ & w940 & EBSD & $1000 \times 500$ & 86.5 & 95.6 & 0.5 & 8 misors & 2 & 0.3 & euoz-th25-itjitji-x-1y-1 & 1.2 \\
\hline $1 b$ & w1092 & EBSD & $1100 \times 1400$ & 76 & 89.8 & 0.5 & 8 misors & 2 & 0.3 & eoz-th50mjtji & 2.5 \\
\hline $1 b$ & w1092-s30 & EBSD & $483 \times 904$ & 77.3 & 92.9 & 0.5 & 8 misors & 2 & 0.3 & eoz-th50mjitji & 2.5 \\
\hline $2 \mathrm{a}$ & w1086 & EBSD & $1200 \times 400$ & 72 & 81 & 0.5 & 8 misors & 2 & 0.3 & eoz-th25-er5-mmmjitji & 1.2 \\
\hline $2 b$ & w946 & EBSD & $1500 \times 970$ & 94.3 & 98.6 & 0.5 & 8 misors & 2 & 0.3 & eoz-th50itji & 2.5 \\
\hline $3 a$ & w1010-s34 & EBSD & $450 \times 980$ & 82.1 & 91.2 & 1 & 8 misors & 2 & 0.5 & eoz-th50i-tjitji & 2.5 \\
\hline $3 a$ & w1010-s36 & EBSD & $500 \times 830$ & 78.5 & 90 & 1 & 8 misors & 2 & 0.5 & eoz-th50i-tjitji & 2.5 \\
\hline $3 b$ & w935 & EBSD & $1277 \times 1027$ & 92.3 & 95.6 & 1 & 8 misors & 2 & 0.5 & eoz-th32i-e5dH-mjitji & 1.5 \\
\hline $3 \mathrm{c}$ & w965-s40 & EBSD & $840 \times 700$ & 76.9 & 88.6 & 1 & 8 misors & 4 & 0.3 & ueuoz-th50er5-ttjitji & 2.5 \\
\hline $3 c$ & w965-s45 & EBSD & $720 \times 1600$ & 89 & 94.8 & 0.3 & 8 misors & 1 & 0.3 & eoz+m-th40-e5dG-mjitji & 2 \\
\hline
\end{tabular}

Table A3. Distribution fitting.

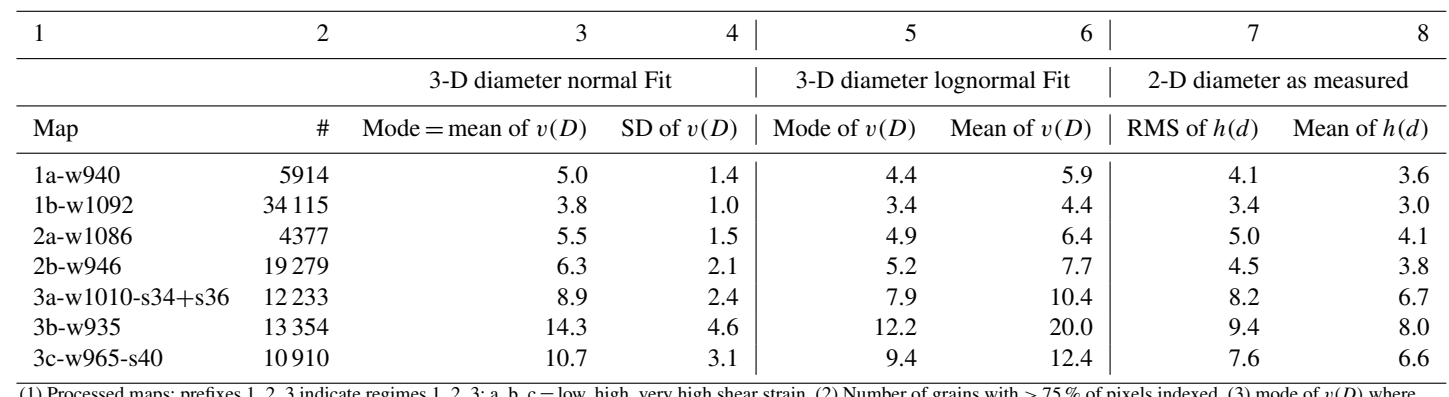

(1) Processed maps: prefixes 1, 2, 3 indicate regimes 1, 2, 3; a, b, c $=$ low, high, very high shear strain. (2) Number of grains with $>75 \%$ of pixels indexed, (3) mode of $v(D)$ where $v=$ volume weighted distribution and $D=$ diameter of recalculated 3-D grains (volume equivalent spheres) = mean of Gaussian fit, (4) SD of Gauss fit, (5) mode calculated from lognormal fit, (6) mean calculated from lognormal fit, (7) root mean square of frequency distribution of diameter of 2-D sections $h(d)$ where $h=$ number density and $d=$ diameter of 2-D grains (area equivalent circles), (8) mean of frequency distribution of diameter of 2-D sections $h(d)$. 


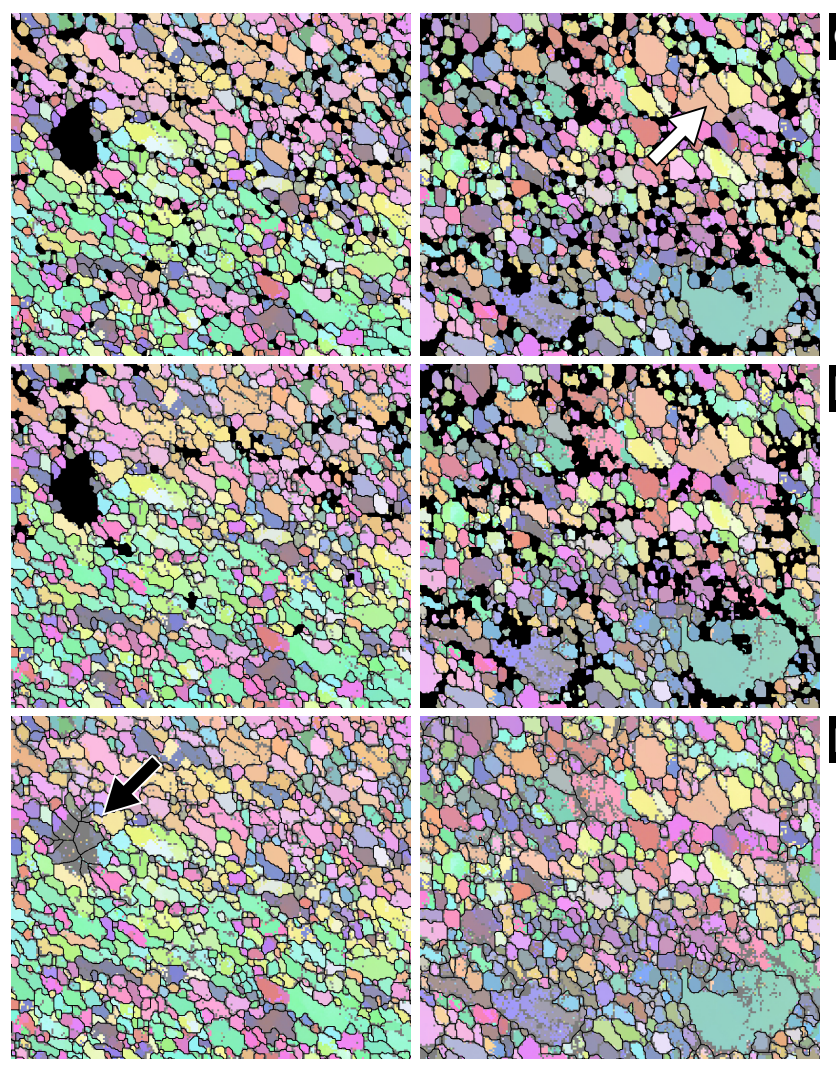

CIP outlines $>75 \%$
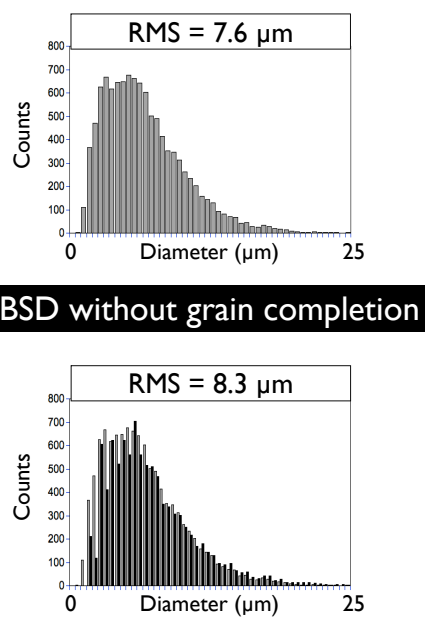

EBSD with grain completion

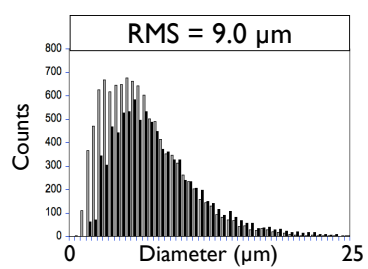

Figure A1. Segmentation based on texture. Comparison of segmentations based on $c$-axis misorientation and structural filtering (CIP) and on full-texture misorientation (EBSD) with and without grain completion (see text). For each segmentation method, two grain boundary maps are shown superposed on a soft Euler RGB image; on the left an area with relatively low (78\%) indexing ratio, on the right an area with relatively high (94\%) indexing ratio. The white arrow points to a low-angle grain boundary that is detected through structural filtering. The black arrow points to a segmentation artifact. On the right are frequency distributions, $h(d)$, where $d=$ diameter of the area equivalent circle. Black bars represent EBSD segmentation and gray bars represent CIP segmentation; root mean square (RMS) values of $h(d)$ are indicated.

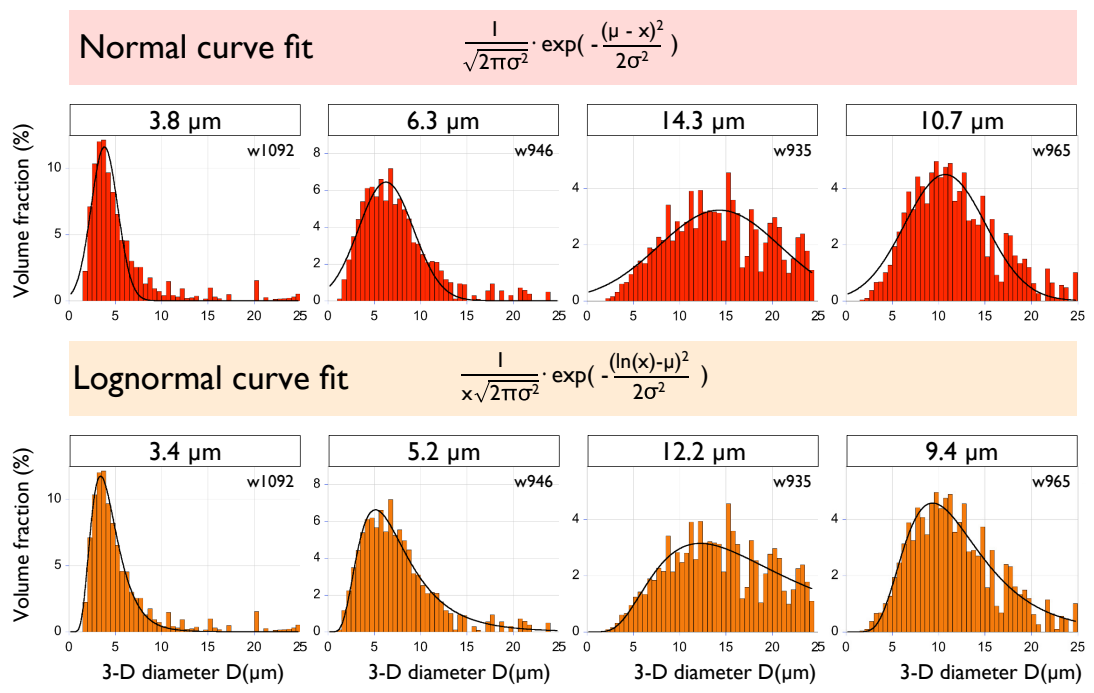

Figure A2. Deriving modal grain size. Volume-weighted histograms $v(D)$ are shown for four high-strain samples of regimes 1, 2, and 3. To derive the modal value, a normal (Gaussian) and a lognormal curve fit are used. The derived modal values are indicated. 


\section{The Supplement related to this article is available online at https://doi.org/10.5194/se-8-1071-2017-supplement.}

Competing interests. Renée Heilbronner is a member of the editorial board of the journal.

Special issue statement. This article is part of the special issue "Analysis of deformation microstructures and mechanisms on all scales". It is a result of the EGU General Assembly 2016, Vienna, Austria, 17-22 April 2016.

Acknowledgements. We are indebted to Jan Tullis who not only provided the samples of this study but who continues to contribute, in the generous fashion typical for her, to the advancement of microstructure and rock deformation studies. We wish to thank Willy Tschudin of Basel University for the preparation of excellent thin sections and Tom Eilertsen and Kai Neufeld of the Arctic University of Troms $\emptyset$ for their dedicated technical support and the time and effort spent during the acquisition of the EBSD maps. Michael Bestmann is thanked for always sharing his immense experience. The paper has further profited from discussions with Michael Stipp, Greg Hirth, and Andreas Kronenberg. Support by the National Science Foundation of Switzerland (grant no. NF 200021-138216; Deformation mechanisms in naturally and experimentally deformed minerals and rocks) is gratefully acknowledged.

Edited by: Bernhard Grasemann

Reviewed by: two anonymous referees

\section{References}

Bachmann, F., Hielscher, R., and Schaeben, H.: Grain detection from $2 d$ and $3 d$ EBSD data-Specification of the MTEX algorithm, Ultramicroscopy, 111, 1720-1733, https://doi.org/10.1016/j.ultramic.2011.08.002, 2011.

Bilby, B. A. and Kolbuszewski, M. L.: Finite deformation of an inhomogeneity in 2-dimensional slow viscous incompressibleflow, P. Roy. Soc. Lond. A Mat., 355, 335-353, 1977.

Bouchez, J. L. and Pecher, A.: The Himalayan main central thrust pile and its quartz-rich tectonites in Central Nepal, Tectonophysics, 78, 23-50, 1981.

Chernak, L. J., Hirth, G., Selverstone, J., and Tullis, J.: Effect of aqueous and carbonic fluids on the dislocation creep strength of quartz, J. Geophys. Res.-Sol. Ea., 114, B04201, https://doi.org/10.1029/2008JB005884, 2009.

DellAngelo, L. and Tullis, J.: Fabric development in experimentally sheared quartzites, Tectonophysics, 169, 1-21, 1989.

Fossen, H. and Tikoff, B.: The deformation matrix for simultaneous simple shearing, pure shearing and volume change, and its application to transpression transtension tectonics, J. Struct. Geol., 15, 413-422, 1993.
Gay, N.: Pure shear and simple shear deformation of inhomogeneous viscous fluids, 1. Theory, Tectonophysics, 5, 211-234, 1968.

Gleason, G. C., Tullis, J., and Heidelbach, F.: The role of dynamic recrystallization in the development of lattice preferred orientations in experimentally deformed quartz aggregates, J. Struct. Geol., 15, 1145-1168, 1993.

Heilbronner, R. and Barrett, S.: Image Analysis in Earth Sciences, Springer, Berlin, Heidelberg, 2014.

Heilbronner, R., and Tullis, J.: The effect of static annealing on microstructures and crystallographic preferred orientations of quartzites experimentally deformed in axial compression and shear, in: Deformation Mechanisms, Rheology and Tectonics: Current Status and Future Perspectives, edited by: DeMeer, S., Drury, M., DeBresser, J., and Pennock, G., Geol. Soc. Sp., 200, 191-218, https://doi.org/10.1144/GSL.SP.2001.200.01.12, 2002.

Heilbronner, R. and Tullis, J.: Evolution of c axis pole figures and grain size during dynamic recrystallization: Results from experimentally sheared quartzite, J. Geophys. Res.-Sol. Ea., 111, B10202, https://doi.org/10.1029/2005JB004194, 2006.

Hielscher, R. and Schaeben, H.: A novel pole figure inversion method: specification of the MTEX algorithm, J. Appl. Crystallogr., 41, 1024-1037, https://doi.org/10.1107/S0021889808030112, 2008.

Hirth, G. and Tullis, J.: Dislocation creep regimes in quartz aggregates, J. Struct. Geol., 14, 145-159, 1992.

Holyoke III, C. W. and Kronenberg, A. K.: Accurate differential stress measurement using the molten salt cell and solid salt assemblies in the Griggs apparatus with applications to strength, piezometers and rheology, Tectonophysics, 494, 17-31, https://doi.org/10.1016/j.tecto.2010.08.001, 2010.

Kidder, S., Hirth, G., Avouac, J.-P., and Behr, W.: The influence of stress history on the grain size and microstructure of experimentally deformed quartzite, J. Struct. Geol., 83, 194-206, https://doi.org/10.1016/j.jsg.2015.12.004, 2016.

Kilian, R. and Heilbronner, R.: Texture analysis of experimentally deformed Black Hills Quartzite, Solid Earth Discuss., https://doi.org/10.5194/se-2017-44, in review, 2017.

Law, R. D.: Deformation thermometry based on quartz c-axis fabrics and recrystallization microstructures: A review, J. Struct Geol., 66, 129-161, https://doi.org/10.1016/j.jsg.2014.05.023, 2014.

Lopez-Sanchez, M. A. and Llana-Funez, S.: An extension of the Saltykov method to quantify 3D grain size distributions in mylonites, J. Struct. Geol., 93, 149-161, https://doi.org/10.1016/j.jsg.2016.10.008, 2016.

Mancktelow, N. S.: Atypical textures in quartz veins from the Simplon fault zone, J. Struct. Geol., 9, 995-1005, 1987.

Mancktelow, N. S.: Deformation of an elliptical inclusion in twodimensional incompressible power-law viscous flow, J. Struct. Geol., 33, 1378-1393, https://doi.org/10.1016/j.jsg.2011.06.005, 2011.

Paterson, M. S. and Olgaard, D. L.: Rock deformation tests to large shear strains in torsion, J. Struct. Geol., 22, 1341-1358, 2000.

Pec, M., Stunitz, H., Heilbronner, R., and Drury, M.: Semi-brittle flow of granitoid fault rocks in experiments, J. Geophys. Res. 121, 1677-1705, https://doi.org/10.1002/2015JB012513, 2016.

Pennacchioni, G., Menegon, L., Leiss, B., Nestola, F., and Bromiley, G.: Development of crystallographic preferred orientation 
and microstructure during plastic deformation of natural coarsegrained quartz veins, J. Geophys. Res.-Sol. Ea., 115, B12405, https://doi.org/10.1029/2010JB007674, 2010.

Poirier, J.: Creep of Crystals: High-Temperature Deformation Processes in Metals, Ceramics and Minerals, Cambridge Univ. Press, Cambridge, 1985.

Poirier, J. and Guillope, M.: Deformation induced recrystallization of minerals, B. Mineral., 102, 67-74, 1979.

Post, A., Tullis, J., and Yund, R.: Effects of chemical environment on dislocation creep of quartzite, J. Geophys. Res.-Sol. Ea., 101, 22143-22155, 1996.

Rahl, J. M. and Skemer, P.: Microstructural evolution and rheology of quartz in a mid-crustal shear zone, Tectonophysics, 680, 129139, https://doi.org/10.1016/j.tecto.2016.05.022, 2016.

Ranalli, G.: Rheology of the Earth, 1st Edn., Allen and Unwin, London, 1987.

Richter, B., Stunitz, H., and Heilbronner, R.: Stresses and pressures at the quartz-to-coesite phase transformation in shear deformation experiments, J. Geophys. Res.-Sol. Ea., 121, 8015-8033, https://doi.org/10.1002/2016JB013084, 2016.

Schmid, S. and Casey, M.: Complete fabric analysis of some commonly observed quartz $c$-axis patterns, in: Geophysical Monograph, Vol. 36, American Geophysical Union, Washington D.C., 263-286, 1986.

Schmid, S., Panozzo, R., and Bauer, S.: Simple shear experiments on calcite rocks - rheology and microfabric, J. Struct. Geol., 9, 747-778, 1987.

Shigematsu, N., Prior, D. J., and Wheeler, J.: First combined electron backscatter diffraction and transmission electron microscopy study of grain boundary structure of deformed quartzite, J. Microsc.-Oxford, 224, 306-321, 2006.
Shimizu, I.: Theories and applicability of grain size piezometers: The role of dynamic recrystallization mechanisms, J. Struct. Geol., 30, 899-917, https://doi.org/10.1016/j.jsg.2008.03.004, 2008.

Stipp, M. and Kunze, K.: Dynamic recrystallization near the brittle-plastic transition in naturally and experimentally deformed quartz aggregates, Tectonophysics, 448, 77-97, https://doi.org/10.1016/j.tecto.2007.11.041, 2008.

Stipp, M. and Tullis, J.: The recrystallized grain size piezometer for quartz, Geophys. Res. Lett., 30, 2088, https://doi.org/10.1029/2003GL018444, 2003.

Stipp, M., Stunitz, H., Heilbronner, R., and Schmid, S.: The eastern Tonale fault zone: a "natural laboratory" for crystal plastic deformation of quartz over a temperature range from 250 to $700^{\circ}$ C, J. Struct. Geol., 24, 1861-1884, 2002.

Tullis, J.: Preferred orientation of quartz produced by slip during plane strain, Tectonophysics, 39, 87-102, https://doi.org/10.1016/0040-1951(77)90090-7, 1977.

Tullis, J., Christie, J., and Griggs, D.: Microstructures and preferred orientations of experimentally deformed quartzites, Geol. Soc. Am. Bull., 84, 297-314, 1973.

Twiss, R.: Theory and applicability of a recrystallized grain-size paleopiezometer, Pure Appl. Geophys., 115, 227-244, 1977. 